\title{
Assessment of Measurement Distortions in GNSS Antenna Array Space-Time Processing
}

\author{
Thyagaraja Marathe, Saeed Daneshmand, and Gérard Lachapelle \\ PLAN Group, Department of Geomatics Engineering, Schulich School of Engineering, University of Calgary, \\ 2500 University Drive NW, Calgary, AB, Canada T2N1N4
}

Correspondence should be addressed to Thyagaraja Marathe; tmarathe@ucalgary.ca

Received 15 November 2015; Revised 12 January 2016; Accepted 20 January 2016

Academic Editor: Lei Yu

Copyright (C) 2016 Thyagaraja Marathe et al. This is an open access article distributed under the Creative Commons Attribution License, which permits unrestricted use, distribution, and reproduction in any medium, provided the original work is properly cited.

\begin{abstract}
Antenna array processing techniques are studied in GNSS as effective tools to mitigate interference in spatial and spatiotemporal domains. However, without specific considerations, the array processing results in biases and distortions in the cross-ambiguity function (CAF) of the ranging codes. In space-time processing (STP) the CAF misshaping can happen due to the combined effect of space-time processing and the unintentional signal attenuation by filtering. This paper focuses on characterizing these degradations for different controlled signal scenarios and for live data from an antenna array. The antenna array simulation method introduced in this paper enables one to perform accurate analyses in the field of STP. The effects of relative placement of the interference source with respect to the desired signal direction are shown using overall measurement errors and profile of the signal strength. Analyses of contributions from each source of distortion are conducted individually and collectively. Effects of distortions on GNSS pseudorange errors and position errors are compared for blind, semi-distortionless, and distortionless beamforming methods. The results from characterization can be useful for designing low distortion filters that are especially important for high accuracy GNSS applications in challenging environments.
\end{abstract}

\section{Introduction}

Satellite based navigation systems are now widely used for positioning and timing. Signals from different Global Navigation Satellite System (GNSS) constellations (e.g., GPS, GLONASS, which are globally operational; BeiDou, Galileo, which are scheduled to be fully operational; IRNSS, QZSS, which provide regional coverage) can offer increased accuracy, robustness, availability, and reliability [1]. Even though GNSS offers superior and diverse features, the position, velocity, and time (PVT) estimates provided by a receiver can degrade due to the presence of interfering signals. Hence, interference is a concern to both civil and military GNSS communities with an increasing focus towards safe and secure applications. Interference affects primarily signal acquisition and tracking and also degrades navigation parameter estimation. By using antenna arrays in GNSS receivers, the spatial and temporal characteristics of the incident signals can be exploited to mitigate interference. Research into antenna array-based methods for GNSS interference mitigation has begun several years ago. Advanced receivers that use Controlled Radiation Pattern Antennas (CRPA) are able to dynamically change the gain patterns and spatially filter RF interference $[2,3]$. Further enhancement can be provided by including a Tapped Delay Line (TDL) behind each antenna element and by performing space-time processing (STP).

STP combines information available from both spatial and temporal domains and can be used in receivers to mitigate both narrowband and wideband interference while preserving the GNSS signals. Compared to space-only processing, the enhancement achieved is mainly due to an increase in the array's degree of freedom [4]. However, this mitigation technique may deteriorate signal acquisition and tracking performance and degrade the signals by introducing some distortions [5]. In coarse acquisition (C/A) code phase based GNSS receivers, pseudorange measurements are generated from the cross correlation function (CCF), which is the time domain form of the cross-ambiguity 
function (CAF). In a receiver, local replica of the $\mathrm{C} / \mathrm{A}$ code is generated to track the incoming signal continuously. The peak tracking value (prompt $(P)$ value) of the correlator is decided based on the shifted local replicas (i.e., Early (E) and Late (L) correlator arms). For proper code tracking, the midpoint between $\mathrm{E}$ and $\mathrm{L}$ arms gives the location of the peak $(P)[6,7]$. A nonlinear behavior in the phase response of the space-time filter may result in distorted CCFs and biased pseudorange measurements [8]. In general, spacetime processing does not provide a linear phase frequency response across the operating band due to the architecture of the space-time filter [9]. In contrast to the other fields such as Radar and wireless applications (e.g., signal-to-noise ratio (SNR) based methods), it is critical to reduce the array processing borne distortions to maintain error-free pseudoranges because erroneous values have a direct impact on achievable accuracy and integrity requirements $[8,10,11]$. Some efforts to characterize and reduce these distortions have been completed [10, 12]. Fante and Vaccaro [10] characterized the distortions on CCF and its widening for interference and multipath conditions. Distortions on the CCF for a sevenelement antenna array in the presence of two interferers were characterized by Myrick et al. [13]. However, effects of the distortions in the position domain were not evaluated.

In general, beamformer is a processor that performs filtering in the spatial (or spatial-temporal) domain by linearly combining spatially (or spatially and temporally) sampled data from each antenna [14]. Considering distortions as a criterion, beamformers can be classified as blind, semidistortionless, and distortionless [15]. In case of blind beamformers, the angles of arrival (AoA) of satellite signals are not used while designing a space-time filter; achieving the interference cancellation while maintaining a linear phase of the filter might be challenging. In such cases, the phase delays for different satellite signals through the space-time filter can be different and these delays introduce biases in the pseudorange measurements. Furthermore, occurrences of the unintentional nulls may affect receiver performance. Semi-distortionless STP methods employ steering vectors that use AoA to avoid unintentional nulls and reduce the distortions $[5,13,16]$. Nonetheless, due to the lack of explicit assumption on the linearity of space-time filter response, these methods do not guarantee distortionless responses for GNSS signals. A few other proposed methods effectively reduce the induced bias errors $[8,17]$; however, they do not guarantee phase linearity. In the distortionless methods, not only the steering vectors are incorporated, but also the filters are designed to have a linear phase or zero phase and theoretically provide a distortionless response $[10,18]$. In one approach, an additional filter is cascaded with the original filter. The frequency response of this filter is the conjugate of frequency response of the space-time filter and therefore the resulting frequency response is real and zero phase [10]. In another approach, filter coefficients are designed such that the filter is linear phase and at the same time interference is suppressed [18]. It is conclusive from these discussions that attempts are done to minimize the distortions by combining the information from steering vectors and by forcing the filter to have linear phase frequency response. The magnitude of the filter-induced biases depends on many factors, including the beamforming method and the angle of incidence of the interference source [17]. Bias introduced in the code phase measurements due to array processing can be in the order of meters in simple interference scenarios to several hundred meters in harsh interference environments $[15,17,19,20]$. Hence, it is important to understand the characteristics of the biases introduced during array processing to assess the quality of the measurements in interference scenario.

The methodology used for simulating GPS signals and interference on the antenna array is described in Section 2. The system model, theoretical analysis of the distortions after STP, and the mathematical formulation for different beamformers used in this research and the assumptions made are discussed in Section 3. In Section 4, the following results from simulations are presented and analyzed: effects of the placement of the interference source with respect to satellite signal, effects due to noise and intersatellite signal cross correlation, and comparison of distortions in blind, semi-distortionless, and distortionless beamformers. The description of the setup used for live data collection using real antenna array and discussion and analysis of the results corresponding to these experiments are given in Section 5.

\section{Simulation Methodology}

Due to regulations, outdoor radio frequency (RF) power transmission in the GNSS bands is prohibited. Therefore, to undertake research involving GNSS signals impacted by interference from jammers and other transmitters, one should ideally have access to outdoor test facilities like GATE (Galileo Test and Development Environment) [21] or an anechoic chamber. An alternative way to generate interference is to combine interference signals through wires while collecting GNSS signals. However, the data collection setup would be complex and require many combiners, cables, and connectors. This would also pose a limitation on incorporating the spatial model parameters (required for multiantenna applications) like the AoA of GNSS and interference signals; hence, full control on the simulation environment would not be present. The hardware simulators available on the market have mostly single antenna simulation capability and adding multiantenna capability is complex and expensive. Therefore, a GNSS software simulator capable of generating both interference and satellite signals corresponding to an antenna array is preferred for evaluating interference mitigation methods. Considering the above, distortions in STP are characterized herein mainly by using an antenna array simulation test bed, which is developed based on the accurate data recorded from a single antenna hardware simulator. This simulation platform enables one to evaluate the different aspects of distortions due to STP.

For GNSS applications, Dong [22] proposed a single antenna GPS signal simulation method. An approach to generate data samples for space-time adaptive processing in GPS is discussed by Zhao et al. [23]. However, as seen in the literature, the majority of GNSS software simulations are performed by considering the carrier and C/A code $[23,24]$. 


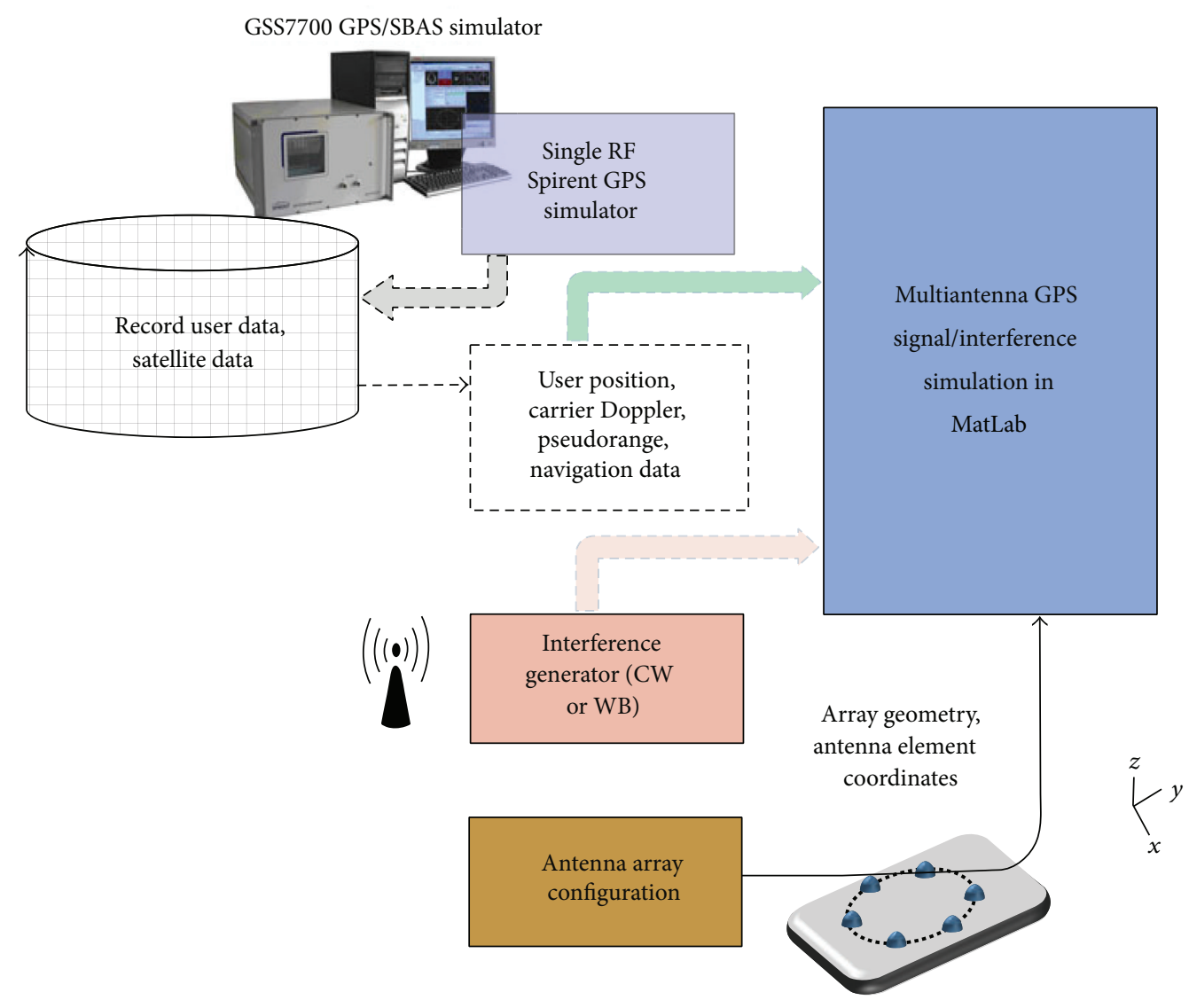

FIGURE 1: Multiantenna GPS-interference simulation scheme.

A sample navigation data is added in a few simulations. All signal processing related results can be analyzed with these methods. However, since the actual navigation data is not embedded, evaluation cannot be done in the position domain.

In this paper, GPS L1 C/A code is used for simulations and analyses; the methods and analyses presented here are applicable to other signals. A simulation test bed is developed to obtain the GPS scenarios and simulate signals as received by an antenna array. Moreover, the scope of the simulations includes the generation of interference signals like continuous wave (CW)/wideband (WB) jammers, which are then added to the generated GPS signals.

Whenever GPS signals on an antenna array are to be simulated, one should know the signal characteristics and the antenna array configurations a priori. The signal characteristics are obtained using a single antenna hardware simulator, namely, the Spirent GSS-7700 simulator in this case [25]. Array configuration and the physical arrangement of the array elements are preset by the user. The steps involved in the proposed signal simulation scheme are shown in Figure 1. Corresponding to the simulation start time and desired total run duration, the user position and satellite positions are recorded and obtained from the simulator. The specific signal parameters like the carrier Doppler, pseudorange, code offset, satellite position, and navigation data are also derived from the simulator data log files. This enables precise and accurate multiantenna scenario generation to maintain high fidelity. Since the exact locations of the satellites are known a priori, precise steering vectors can be generated.

GPS Signal Simulation for a Single Antenna. The signal model of the GPS signal received at the user receiver antenna for the $p$ th satellite can be modelled as

$$
\begin{aligned}
s_{p}(t)= & \sqrt{2 C_{p}} \cdot d_{p}\left(t-\tau_{p}\right) \cdot c_{p}\left(t-\tau_{p}\right) \\
& \cdot \cos \left(2 \pi\left(f_{p}^{\mathrm{RF}}+f_{p}^{d}\right) t+\varphi_{p}\right)
\end{aligned}
$$

where $\sqrt{2 C_{p}}$ is the amplitude of the generated signal, $d_{p}(t-$ $\left.\tau_{p}\right)$ is the navigation data, $c_{p}\left(t-\tau_{p}\right)$ is the C/A code, $\tau_{p}$ is the offset, $f_{p}^{\mathrm{RF}}$ is the carrier frequency, $f_{p}^{d}$ is the carrier Doppler frequency, and $\varphi_{p}$ is the carrier phase.

GPS Signal Simulation for an Antenna Array. The simulated signals at each of the antenna array elements are distinct in phase, even though the navigation data, carrier, and C/A code parameters are the same. This is because the array geometry and the signal characteristics vary as a function of antenna separation and the AoA of the signals. 
Consider an antenna array with $N$ elements. The signal model for the GNSS and interference-combined environment (i.e., composite form of baseband signal vector) for the antenna array is given as

$$
\underset{N \times 1}{\mathbf{x}}=\left[\begin{array}{c}
x_{1} \\
x_{2} \\
\vdots \\
x_{N}
\end{array}\right]=\sum_{p=1}^{P} \mathbf{C}^{M} s_{p} \underset{N \times 1}{\mathbf{a}_{p}}+\sum_{l=1}^{L} \mathbf{C}^{M} \underset{i_{l} \mathbf{b}_{l}}{ }+\underset{N \times 1}{\boldsymbol{\eta}},
$$

where the first, second, and third part of the right hand side represent the GPS signals, interference, and noise, respectively, $\mathbf{a}_{p}$ and $\mathbf{b}_{l}$ are the respective steering vectors (which includes the spatial information of the signal to be simulated for each element of array) for GPS and interference, and $P$ and $L$ represent the number of satellites and interfering sources, respectively. $\boldsymbol{\eta}$ is the white Gaussian noise vector and $x_{n}, n=1, \ldots, N$ indicates the received signal at the $n$th antenna element. $\mathbf{C}^{M}$ is the mutual coupling matrix which can be represented as

$$
\underset{N \times N}{\mathbf{C}^{M}}=\left[\begin{array}{cccc}
c_{1,1}^{M} & c_{1,2}^{M} & \cdots & c_{1, N}^{M} \\
c_{2,1}^{M} & c_{2,2}^{M} & \cdots & c_{2, N}^{M} \\
\vdots & \vdots & \cdots & \vdots \\
c_{N, 1}^{M} & c_{N, 2}^{M} & \cdots & c_{N, N}^{M}
\end{array}\right]
$$

The diagonal elements of $\mathbf{C}^{M}$ model the differences in amplitude and phase due to unequal cable lengths and other electronic parts and its off-diagonal elements represent the coupling coefficients between the antennas. For simulations, the mutual coupling matrix is considered an identity matrix and for real data collection coupling effects are compensated during antenna array calibration.

Let $\tau_{n}$ be the time delay between the reference antenna element and the $n$th array element, for $n=0,1,2, \ldots,(N-$ 1) with $\tau_{0}=0$. GPS signal frequency for L1 carrier is $1575.42 \mathrm{MHz}$; the signal bandwidth is small (generally, $2 \mathrm{MHz}$ to $20 \mathrm{MHz}$ ). Since bandwidth of GPS envelope is small,

$$
\begin{aligned}
s_{p}\left(t-\tau_{n}\right)= & \sqrt{2 C_{p}} \cdot d_{p}\left(t-\tau_{p}-\tau_{n}\right) \cdot c_{p}\left(t-\tau_{p}-\tau_{n}\right) \\
& \cdot \cos \left(2 \pi\left(f_{p}^{\mathrm{RF}}+f_{p}^{d}\right)\left(t-\tau_{n}\right)+\varphi_{p}\right)
\end{aligned}
$$

can be approximated as

$$
\begin{aligned}
s_{p}\left(t-\tau_{n}\right) \simeq & \sqrt{2 C_{p}} \cdot d_{p}\left(t-\tau_{p}\right) \cdot c_{p}\left(t-\tau_{p}\right) \\
& \cdot \cos \left(2 \pi\left(f_{p}^{\mathrm{RF}}+f_{p}^{d}\right)\left(t-\tau_{n}\right)+\varphi_{p}\right),
\end{aligned}
$$

such that $B_{s} \cdot \Delta T_{\max } \ll 1$, where $B_{s}$ is the maximum envelope bandwidth and $\Delta T_{\max }$ is the maximum time required for the signal to traverse the array [26].

With the approximation in (5), which is also known as narrowband assumption, signal delays are approximated by phase shifts and the array steering vector, which is a function of the signal's AoA, carrier frequency, and the array configuration, is given by

$$
\underset{N \times 1}{\mathbf{a}_{p}}=\left[\begin{array}{c}
e^{j\left(2 \pi / \lambda_{c}\right)\left(\hat{\mathbf{e}}_{p}^{T} \mathbf{d}_{1}^{\mathrm{ant}}\right)} \\
e^{j\left(2 \pi / \lambda_{c}\right)\left(\hat{\mathbf{e}}_{p}^{T} \mathbf{d}_{2}^{\text {ant }}\right)} \\
\vdots \\
e^{j\left(2 \pi / \lambda_{c}\right)\left(\hat{\mathbf{e}}_{p}^{T} \mathbf{d}_{N}^{\text {ant }}\right)}
\end{array}\right],
$$

where $\lambda_{c}$ is the carrier wavelength and $\mathbf{d}_{j}^{\text {ant }}$ is the antenna coordinates vector. $\widehat{\mathbf{e}}_{p}$ is a vector pointing in the direction of the desired signal and is given by

$$
\widehat{\mathbf{e}}_{p}=\left[\begin{array}{c}
\cos \left(\theta_{\mathrm{El}}^{p}\right) \sin \left(\phi_{\mathrm{Az}}^{p}\right) \\
\cos \left(\theta_{\mathrm{El}}^{p}\right) \cos \left(\phi_{\mathrm{Az}}^{p}\right) \\
\sin \left(\theta_{\mathrm{El}}^{p}\right)
\end{array}\right],
$$

where $\theta_{\mathrm{El}}^{p}$ is the elevation angle of the $p$ th signal direction (measured from the horizon to the zenith) and $\phi_{\mathrm{Az}}^{p}$ is the azimuth angle of the $p$ th signal direction.

Validation of the Simulation Test Bed. Fidelity of the simulated signals was assessed using signal tracking metrics like carrier Doppler and $C / N_{0}$, and these were compared with the tracking metrics for commercially available hardware simulators (Spirent GSS-7700 and Rohde and Schwarz SMBV-100 [27]). It was observed that tracking and the positioning performance for signals from the new software simulator match the performance of hardware simulators. The capability of the simulator to generate antenna array signals was validated using the Minimum Power Distortionless Response (MPDR) beamformer [26] and an improvement in the processing gain was observed. The results corresponding to simulator validation are not included in the paper.

Assumptions. The following assumptions were made for signal simulation: (a) there is uniform propagation in all directions of isotropic and nondispersive medium; (b) for far field array processing, the radius of propagation is much greater than the size of the array and there is plane wave propagation; (c) there is a zero mean white noise, which shows lack of correlation (i.e., the noise term in (2) is spatially and temporally white zero mean complex vector with covariance matrix $\sigma_{N}^{2} \mathbf{I}$ ); (d) there is no coupling and the calibration is errorless; and (e) undesired signals (i.e., interference) are considered unknown deterministic signals.

\section{Theoretical Analysis of Distortions and Beamforming Methods}

Distortions are analyzed for the following methods: spacetime blind eigenvector beamformer, MPDR beamformer, extended MPDR (E-MPDR), and cascade distortionless (C$\mathrm{DL})$ beamformer. Space-time eigenvector beamformer is a blind beamformer [28, 29]. The MPDR is a spatial only 


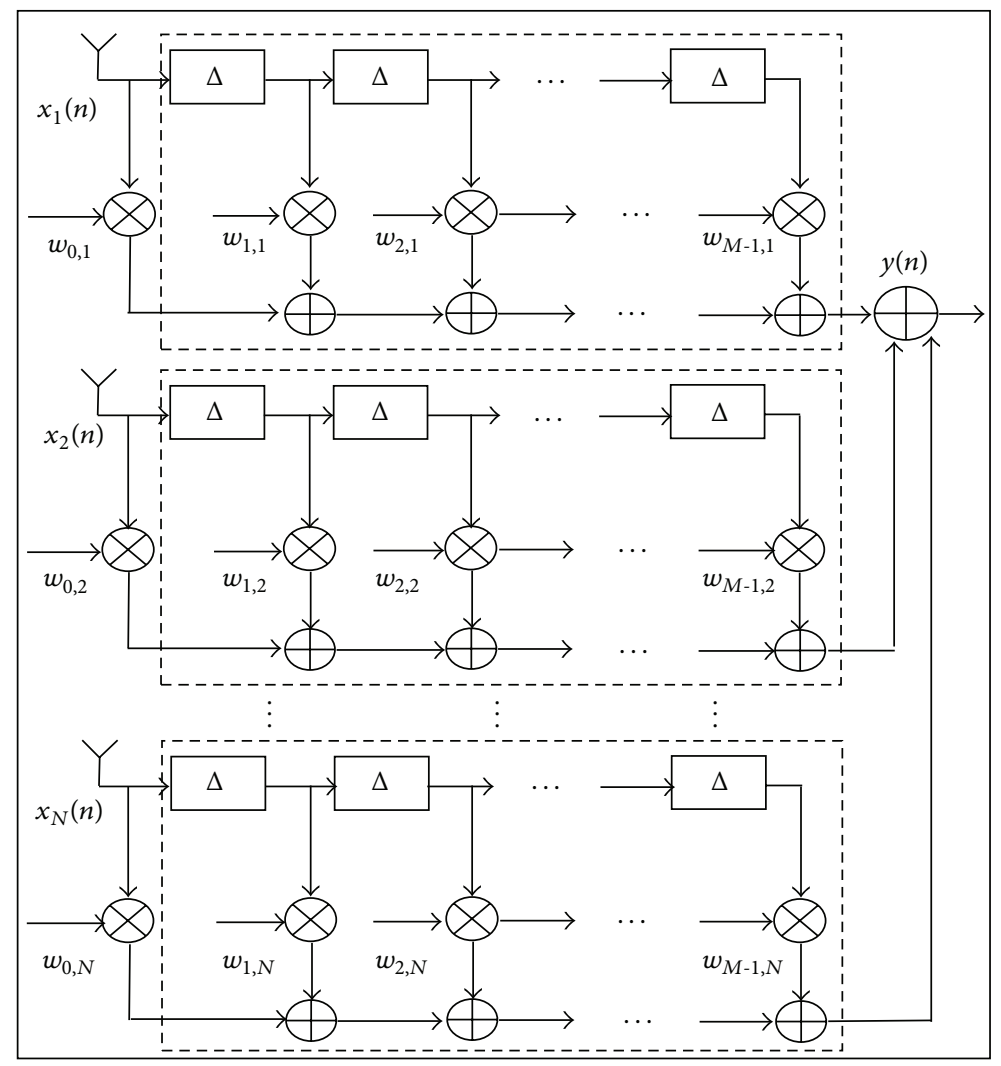

FIgURE 2: Block diagram of the STP.

beamformer that incorporates a steering vector; this beamformer provides a distortionless response for carrier phase measurements [26]. The E-MPDR is an extended version of MPDR for space-time processing to increase the array degrees of freedom (DoF) [15, 30]. Due to the presence of distortions from the temporal filter, this is considered a semi-distortionless method. The C-DL method incorporates satellite steering vectors and is designed to provide zero phase, providing a distortionless filter response [10]. Space-time blind eigenvector, E-MPDR, and C-DL are used in space-time mode and MPDR is used to compare the results for the space-only processing mode (hence, CCFs and position solutions do not experience any distortions due to temporal filtering in MPDR). The system model and the four mentioned beamforming methods are discussed in the sequel.

Theory and System Model. For an antenna array with $N$ elements and $(M-1)$ taps (used for TDLs), NM weights should be computed. A representation of a STP processor for an $N$ element antenna array with $(M-1)$ taps is shown in Figure 2 [31].

At every time epoch, NM samples comprising the antenna elements and the TDLs for a $N M \times 1$ received signal vector are given by

$\overrightarrow{\mathbf{r}}$

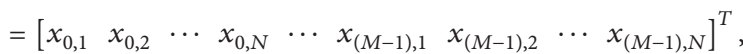

in which $x_{m, n}$ is the $m$ th delayed sample and $m=0,1,2, \ldots$, $(M-1)$ at the $n$th antenna element with range $n=1,2, \ldots, N$. Considering (8), the space-time correlation matrix can be obtained as

$$
\underset{N M \times N M}{\mathbf{R}_{\overrightarrow{\mathbf{r}}}}=E\left\{\overrightarrow{\mathbf{r}}^{H}\right\}
$$

where $E\{\}$ represents the statistical expectation. This correlation matrix is employed in the optimization for different beamforming methods.

3.1. STP Distortions. Interference mitigation using STP allows three types of GNSS signal degradations, namely, CCF misshaping, attenuation, and noise domination, and measurement bias. Firstly, the ideal shape of a typical GNSS signal's CCF is triangular; however, misshaping (asymmetric widening) of the CCF after STP will introduce an error component in the pseudorange measurements. Secondly, when a space-time filter is used for interference mitigation, it is designed to strip off a designated portion of the input (typically interference). Without proper considerations, due to the unintentional nulls being introduced, some portion of the desired signals is attenuated. Based on the magnitude of the attenuation, this may lead to rounding of the CCF tip and a drop in the correlator output value. Thirdly, due to the nonlinearity of the space-time filter, different frequency components (in GNSS, corresponding to Doppler frequencies of different satellite signals) experience different delays 
passing through the filter. Having different biases for different satellite pseudoranges leads to inaccurate position estimates. On the contrary, if the biases are the same across all satellites, it will be absorbed in the receiver clock offset and an accurate position estimate is obtained $[6,7,32]$. Temporal filters in STP can be independently constrained to obtain linear phase by using typical construct of finite impulse response (FIR) filters (i.e., coefficients have conjugate symmetry [33]). Even then, maintaining phase linearity in STP is not easy, as the information from spatial (signals from different antennas) and temporal (delayed signals) domains are combined.

Because of the STP filter structure shown in Figure 2, without special considerations it does not provide a linear phase frequency response across the operating band. Consequently, it is possible that the filter will introduce distortions to the input GPS signals [10]. Fante and Vaccaro [10] and Peng et al. [34] provided a detailed mathematical framework for analyzing the distortions in the GPS CCF. In GPS receivers, the ranging delays are estimated by cross correlation of the received signal with a known signal generated locally. Then the correlation function for the signal after space-time filtering, despreading and Doppler removal can be written as

$$
R(\tau)=\int_{-\infty}^{\infty} H(f) P(f) e^{-j 2 \pi f \tau} d f
$$

where $P(f)$ is the power spectrum of the signal, which is a symmetric function of $f$, and $H(f)$ is the frequency response of the space-time filter defined as $H(f)=\mathbf{h}^{H}(f) \mathbf{a}_{p}$, with $\mathbf{h}(f)$ being the frequency response vector of the TDLs and $\mathbf{a}_{p}$ being the steering vector of the satellite signals. Because of the addition of $H(f)$, the correlator peak can be shifted and the correlator function can be potentially broadened. Only with proper design considerations bias errors and phase shifts can be corrected.

Adding antenna array processing adds biases in code and carrier phase measurements. The phase delays experienced by the signals impinged on different elements of the array can be precisely modelled in the steering vectors. By employing methods such as MVDR or MPDR, the carrier phase delays can be compensated. Hence carrier phase measurements can be bias-free, irrespective of the presence or absence of interference in the data being processed. Conventional MVDR cannot however compensate for code phase based measurements and may cause shifting and widening of the cross correlation function. Depending on the array configuration and signal angle of arrival, this may add measurement biases that can vary between negligibly small values to a few meters; such biases are present in pseudorange measurements that are generated using real antenna arrays. In this paper, array signals are simulated using GPS signals corresponding to a single antenna and then phase translation is performed to obtain signals for other elements of the array. Therefore, code phase pseudoranges are free from biases, as code offsets are not simulated. This simulation method, which uses phase translations, enables one to accurately assess STP filter's temporal distortions.

All types of distortions that show up due to the presence of $H(f)$ in the GPS processing chain as shown in (10) are considered for analysis in this paper.
3.2. Blind Eigenvector Beamformer. In a blind eigenvector beamformer, neither the directions of arrival of the satellite signals nor those of interference signals are used in determining the space-time filter weights and this beamformer is extensively addressed in the literature [18, 26, 28, 29]. The presence of unintentional nulls in this case may cause failure in the acquisition of some satellite signals.

The projection matrix $\mathbf{P}$ (with matrix dimension (NM $L) \times N M$ ) into the interference-free subspace can be obtained by applying an eigenvalue decomposition (EVD) of the correlation matrix $\mathbf{R}_{\overrightarrow{\mathbf{r}}}$ of the input samples as

$$
\begin{aligned}
& \mathbf{R}_{\overrightarrow{\mathbf{r}}} \\
& =\left[\begin{array}{cc}
\mathbf{U}_{\text {Int }} & \mathbf{U}_{\mathrm{Null}} \\
N M \times L & N M \times(N M-L)
\end{array}\right]\left[\begin{array}{cc}
\Lambda_{\mathrm{Int}} & 0 \\
L \times L & \boldsymbol{\Lambda}_{\mathrm{Null}} \\
0 & (N M-L) \times(N M-L)
\end{array}\right]\left[\begin{array}{c}
\mathbf{U}_{\mathrm{Int}}^{H} \\
N M \times L \\
\mathbf{U}_{\mathrm{Null}}^{H} \\
N M \times(N M-L)
\end{array}\right],
\end{aligned}
$$

where $\mathbf{U}_{\text {Int }}$ and $\mathbf{U}_{\text {Null }}$ are the eigenvector matrices of the interference and interference-free signal subspaces, respectively, and $\boldsymbol{\Lambda}_{\text {Int }}$ and $\boldsymbol{\Lambda}_{\mathrm{Null}}$ are the corresponding eigenvalue matrices. By isolating eigenvectors corresponding to the bigger eigenvalues, interference-free subspace (i.e., noiseplus-GNSS signal subspace) can be calculated. Using $\mathbf{P}$ the STP filter weights are computed such that they suppress high power interference (i.e., minimizing the filter's output power) and are given by

$$
\mathbf{w}=\mathbf{P}^{T} \mathbf{g}
$$

where $\mathbf{g}$ is a gain selection vector which can be $\mathbf{1}$ (all one vector) for equal gain combining or can be chosen based on the selection gain combining criteria [35]. Beamforming is performed in the predespreading stage of the receiver where the satellite signal arrival details are still unknown (not extracted). Therefore, a single set of filter weights that works well for all satellites is designed. Looking at the steps involved in this method, it can be seen that no consideration is given towards the satellite signal directions/locations while designing the filter weights. The lack of look direction constraint can result in desired signal distortions.

3.3. Minimum Power Distortionless Response (MPDR) Beamformer. This method incorporates satellite signal steering vectors in the filter as a constrained optimization problem [26]. The optimization problem in this case is defined as

$$
\begin{array}{r}
\mathbf{w}_{\text {opt }}=\underset{\mathbf{w}}{\operatorname{argmin}} \quad\left\{\mathbf{w}^{\mathbf{H}} \mathbf{R} \mathbf{w}\right\}, \\
\text { such that } \quad \mathbf{w}^{H} \mathbf{a}_{p}=1 .
\end{array}
$$

The correlation matrix is constructed only using spatial samples leaving the matrix dimensions as $N \times N$ (only one tap corresponding to zero delay is used). A constraint vector that incorporates AoA is used in finding a solution to this problem. A solution to the above problem is given by

$$
\mathbf{w}_{\mathrm{opt}}=\mathbf{R}^{-1} \mathbf{a}_{p}\left(\mathbf{a}_{p}^{H} \mathbf{R}^{-1} \mathbf{a}_{p}\right)^{-1}
$$


TABLE 1: Comparison of few features offered by beamformers.

\begin{tabular}{|c|c|c|c|c|}
\hline \multirow{2}{*}{ Parameter description } & \multicolumn{4}{|c|}{ Beamforming method } \\
\hline & Blind eigenvector & MPDR & E-MPDR & C-DL \\
\hline Use of steering vector & No & Yes & Yes & Yes \\
\hline Computational complexity & Less & Moderate & High & High \\
\hline Space $(\mathrm{S})$ or space-time $(\mathrm{ST})$ & ST & S & ST & ST \\
\hline Distortions & Maximum & No distortion & Moderate & Minimum \\
\hline Number of filter weight sets & Single & Multiple* & Multiple* & Multiple* \\
\hline
\end{tabular}

* Separate steering vector is used for each satellite.

The optimization problem is solved using the method of Lagrange multipliers [26].

The MPDR method uses signal arrival details $\left(\mathbf{a}_{p}\right)$ in the filter design and consequently all spatial phase differences are compensated. Due to the use of AoA in the constraint of the MPDR, the phase of the GPS signal would not get affected and the signal would pass through the filter undistorted; additionally, due to fact that it is used in a spatial only configuration, it is free from the distortion contributions from temporal filters.

3.4. Extended MPDR (E-MPDR). This is the extended version of the MPDR for space-time processing. Compared to MPDR, this approach provides additional DoF [15]. The optimization problem for the extended MPDR can be expressed as

$$
\mathbf{w}_{\mathrm{opt}}=\underset{\mathbf{w}}{\operatorname{argmin}}\left\{\mathbf{w}^{\mathbf{H}} \mathbf{R} \mathbf{w}\right\},
$$

such that $\mathbf{w}^{H} \mathbf{c}=1$. The vector $\mathbf{c}$ is defined as

$$
\underset{N K \times 1}{\mathbf{c}}=\left[\begin{array}{cccc}
\mathbf{a}_{p}^{T} & \mathbf{0}^{T} & \cdots & \mathbf{0}_{N \times 1}^{T} \\
N \times 1 & & & N \times 1
\end{array}\right]^{T} .
$$

This method incorporates satellite signal steering vectors in the structure of the space-time filter as a constrained optimization problem; consequently, the distortions due to the spatial phase mismatch are reduced. A nonzero constraint is added to the first tap. The filter response is not necessarily a linear phase and as a result it may lead to possible CCF widening and distortion errors, due to the added temporal filters.

3.5. Cascade Distortionless (C-DL) Beamformer. As discussed in Section 3.1, distortions are introduced due to the beamforming/null steering filter. The C-DL method explores the possibility of distortion reduction by cascading an additional filter to achieve linear phase (precisely, zero phase in this method) from the filter [10]. In the filter response $H(f)$, distortions due to spatial processing are reduced by using the steering vectors. However, a linear phase response is not assured. To remove the phase nonlinearity of the spacetime filter, it is cascaded with another filter whose frequency response is the conjugate of the frequency response of the space-time filter. Designing a cascaded filter with proper considerations, symmetric impulse response is obtained that

\begin{tabular}{|c|c|c|c|c|}
\hline PRN & $\mathrm{Az}$ (degree) & El (degree) & Sky plot & \\
\hline 7 & 85 & 11 & & \\
\hline 8 & 78 & 48 & & \\
\hline 10 & 218 & 26 & & \\
\hline 15 & 298 & 50 & 60 & \\
\hline 17 & 146 & 14 & $900^{2}$ & 90 \\
\hline 18 & 332 & 9 & & \\
\hline 19 & 34 & 14 & & \\
\hline 26 & 282 & 69 & & \\
\hline 27 & 57 & 80 & 100 & \\
\hline 28 & 119 & 72 & 180 & \\
\hline
\end{tabular}

TABLE 2: Satellite visibility during test: simulator data.

provides the same shift to all satellite pseudoranges; however, the correlation peak can be broadened. The frequency response of the resulting filter is given as $H(f) H^{*}(f)=$ $|H(f)|^{2}$.

A summary of comparison of the above methods is given in Table 1.

The performance of beamformers depends on the overall information available to them, for example, AoA. Based on the design criteria, the available information is used in the beamformers for interference mitigation to maintain maximum gain in the desired direction, to reduce the distortions, and to maintain linear phase.

\section{Simulation Results and Analyses}

Static GPS data is simulated for six antenna elements placed in a uniform circular fashion with radius equal to half a wavelength (of GPS L1 signals). Each of the simulated interference scenarios has a Jammer-to-Noise Ratio (JNR) of $20 \mathrm{~dB}$ over a bandwidth of $10 \mathrm{MHz}$. A set of GPS satellites providing a good Dilution of Precision (DOP) is chosen for the simulation. Pseudorandom noise (PRN) codes and azimuth and elevation angles of the satellites are given in Table 2. The user is assumed static and the satellite motion and corresponding Doppler changes have been incorporated into the simulations.

Four interference scenarios are considered for the simulations. Table 3 lists different parameters corresponding to the interference sources. CW sources are simulated such that they are present in the GPS L1 main lobe bandwidth. 
TABLE 3: Interference scenarios.

\begin{tabular}{|c|c|}
\hline Scenario & $\begin{array}{l}\text { Parameters description } \\
\text { (angles in degree and frequency in } \mathrm{Hz} \text { ) }\end{array}$ \\
\hline \multirow{4}{*}{ Scenario 1} & One CW interference \\
\hline & Varying azimuth \\
\hline & Varying elevation; details are in Section 4.1 \\
\hline & Frequency offset $=500$ \\
\hline \multirow{4}{*}{ Scenario 2} & One CW interference \\
\hline & Azimuth $=190$ \\
\hline & Elevation $=45$ \\
\hline & Frequency offset $=1500$ \\
\hline \multirow{4}{*}{ Scenario 3} & Two CW interference and one wideband interference \\
\hline & Azimuth $=190,25,40$ \\
\hline & Elevation $=45,30,70$ \\
\hline & Frequency offset $=1500,3500$ \\
\hline \multirow{4}{*}{ Scenario 4} & Six CW interference \\
\hline & Azimuth $=190,25,80,245,320,345$ \\
\hline & Elevation $=45,30,15,40,10,55$ \\
\hline & Frequency offset $=1500,3500,5000,5500,6700,7500$ \\
\hline
\end{tabular}

An open source MatLab ${ }^{\circledR}$ based single antenna software receiver [36] was modified to achieve multiantenna receiver functionality. Acquisition, tracking, and position computation blocks of the original software were modified. One of the antennas is chosen as the reference antenna, and acquisition, tracking, and position estimation are performed using the satellite signals received at that antenna. The local signal replicas corresponding to this antenna path were used to measure the relative amplitude and phase values of the signals at other antennas. Hence, the estimated discriminator outputs at different antennas differ only in amplitude and phase.

Several metrics that indicate performance of the receiver's operation at different stages are used for comparison. Distortions to the CCFs are quantified using a distortion metric, the total error in the GPS pseudoranges due to array processing is measured using overall measurement error (or measurement bias), effective $C / N_{0}$ indicates the quality of code tracking, and the contributions of the measurement errors are observed by comparing the ENU position errors. The number of satellites tracked is given wherever applicable.

Distortion Metric (DM). The metric shown in Figure 3 is used to quantify the distortion in the CCF shape compared to the clean CCF shape [35]. Due to the asymmetric widening of the CCF, the prompt $(P)$ derived using $\mathrm{E}$ and $\mathrm{L}$ arms will have an offset from the $P$ value of the clean signal, leading to GPS measurement errors. The error introduced only due to CCF misshaping is termed a distortion metric (DM) and is measured in meters. Due to asymmetry in widening, the distortion metric depends on the correlator spacing chosen for code tracking. During simulations ideal signal conditions are considered; distortions due to multipath are not present in the metric.

Overall Measurement Error (Measurement Bias). In the CCF distortion metric explained previously, the bias introduced

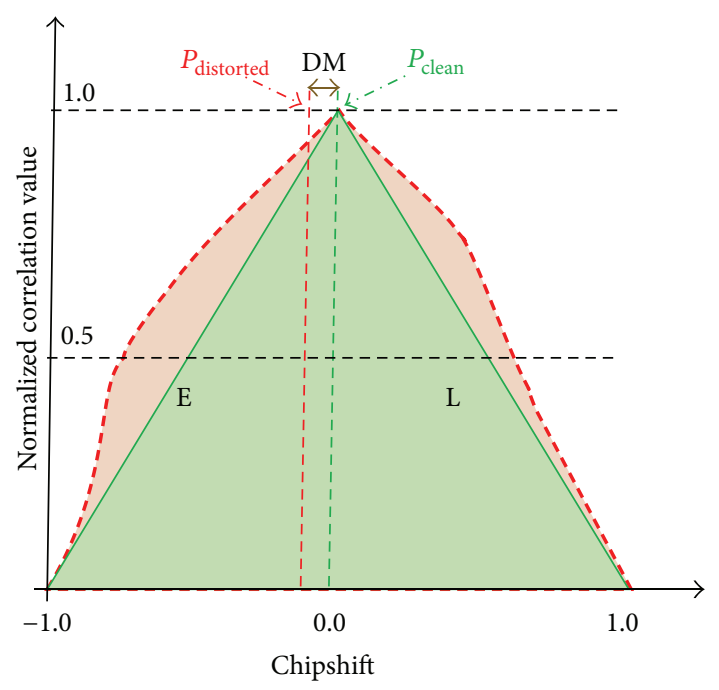

FIGURE 3: CCF distortion metric.

only by CCF misshaping was measured. However, the absolute biases introduced due to space-time processing, which can be same or different for different satellites, were not quantified. These are computed as

$$
\Delta \rho^{p}=\rho_{A P}^{p}-\rho_{c}^{p}
$$

where $\Delta \rho^{p}$ is the measurement bias (in $\mathrm{m}$ ), $\rho_{A P}^{p}$ is the pseudorange measurement corresponding to the chosen array processing method, and $\rho_{c}^{p}$ is the pseudorange measurement for a single RF clean signal (without any interference and without any spatial-temporal processing) for $p$ th satellite. Generally, $\Delta \rho^{p}$ comprises a fixed part $\Delta \rho_{F}^{p}$ and a variable part $\Delta \rho_{V}^{p}$. For a linear phase FIR filter with conjugate symmetric coefficients, the variable part $\Delta \rho_{V}^{p}=0$, and the fixed part can be given by

$$
\Delta \rho_{F}^{p}=T_{s} C\left(\frac{M-1}{2}\right),
$$

where $T_{s}$ is the delay due to one tap (in units of time), $C$ is the speed of light $(\mathrm{m} / \mathrm{s})$, and $M$ is the number of taps used in the temporal filter.

Effective $C / N_{0}$. To measure the effect of interfering signals on the quality of the prompt code tracking channel of a GPS receiver, a metric called effective $C / N_{0}$ is considered and it is calculated based on the following equation [37]:

$$
\begin{aligned}
& \left(\frac{C}{N_{0}}\right)_{\text {eff }} \\
& =\frac{C_{s} \int_{-\beta_{r} / 2}^{\beta_{r} / 2} G_{s}(f) d f}{N_{0} \int_{-\beta_{r} / 2}^{\beta_{r} / 2} G_{s}(f) d f+C_{j} \int_{-\beta_{r} / 2}^{\beta_{r} / 2} G_{j}(f) G_{s}(f) d f},
\end{aligned}
$$

where $C_{s}$ is received power of the desired signal, $G_{s}(f)$ is the normalized power spectral density (PSD) of the desired signal, and $N_{0}$ is the thermal noise power density. 
TABLE 4: Changing interference placement: overall measurement errors (in metres) $\left(\Delta \rho_{V}^{p}\right)$.

\begin{tabular}{cccccccccc}
\hline & & \multicolumn{7}{c}{ Azimuth (degree) } \\
& 0 & 60 & 120 & 180 & 240 & 300 & 30 \\
\hline Elevation (degree) & & & & & & \\
0 & 0.2 & -0.8 & -1.0 & 0.6 & 0.2 & -0.9 & $*$ \\
30 & -0.4 & -0.1 & -0.1 & 0.5 & 0.3 & -1.0 & $*$ \\
60 & 1.2 & 1.6 & 1.2 & 0.0 & 0.0 & 0.0 & $*$ \\
90 & -114 & -114 & -114 & -114 & -114 & -114 & $*$ \\
70 & $*$ & $*$ & $*$ & $*$ & $*$ & $*$ & $*$ \\
85 & $*$ & $*$ & $*$ & $*$ & $*$ & $*$ & $*$ \\
\hline
\end{tabular}

TABLE 5: Changing interference incident angle: $C / N_{0}$ (in $\mathrm{dB}-\mathrm{Hz}$ ).

\begin{tabular}{ccccccccc}
\hline & \multicolumn{9}{c}{ Azimuth (degrees) } \\
& 0 & 60 & 120 & 180 & 240 & 300 & 30 \\
\hline Elevation (degrees) & & & & & & \\
0 & 54.5 & 54.7 & 54.6 & 54.7 & 54.9 & 54.7 & $*$ \\
30 & 54.8 & 54.9 & 54.9 & 54.5 & 54.7 & 54.5 & $*$ & $*$ \\
60 & 54.2 & 52.8 & 54.4 & 55.1 & 55.0 & 55.0 & $*$ \\
90 & 49.5 & 49.5 & 49.5 & 49.5 & 49.5 & 49.5 & $*$ \\
70 & $*$ & $*$ & $*$ & $*$ & $*$ & $*$ & $*$ \\
85 & $*$ & $*$ & $*$ & $*$ & $*$ & $*$ & $*$ \\
\hline
\end{tabular}

$C_{j}$ is the interference power and $G_{j}(f)$ is the normalized PSD of the interference signal. $\beta_{r}$ is the receiver front-end bandwidth. Assuming that the front-end bandwidth is wide enough to pass all the signal and interference frequency components, (19) reduces to

$$
\left(\frac{C}{N_{0}}\right)_{\mathrm{eff}}=\frac{C_{s}}{N_{0}+C_{j} \int_{-\beta_{r} / 2}^{\beta_{r} / 2} G_{j}(f) G_{s}(f) d f} .
$$

Equation (20) can be extended for array processing and the expression for effective $C / N_{0}$ is

$$
\begin{aligned}
& \left(\frac{C}{N_{0}}\right)_{\mathrm{eff}}^{\text {Array }} \\
& \quad=\frac{\left(A_{\mathrm{BF}}\right) C_{s}}{N_{0}+\sum_{l=1}^{L}\left(\left(A_{\mathrm{NS}}\right)_{l} C_{l} \int_{-\beta_{l} / 2}^{\beta_{l} / 2} G_{l}(f) G_{s}(f) d f\right)},
\end{aligned}
$$

where $A_{\mathrm{BF}}$ is the beamforming gain and $\left(A_{\mathrm{NS}}\right)_{l}$ is the loss due to null steering at $l$ th interference signal. $C_{l}$ is the interference power and $G_{l}(f)$ is the normalized PSD of $l$ th interference signal having bandwidth $\beta_{l}$. Effective $C / N_{0}$ for array signals given in (21) will be referred to as $C / N_{0}$ in the sequel.

4.1. Effects Based on Interference Source Incident Angle. Array processing methods primarily process the signals in the spatial domain and the performance of the beamforming process depends on the relative angles between the desired and interference signals. Here, the effects of the interference source incidence angle on receiver performance are evaluated. To analyze the effects of spatial closeness between the desirable and interference signals, a CW interference source is placed at different spatial separations (as per Scenario 1 given in Table 3) from a GPS satellite signal, that is, PRN27 located at an elevation of $80^{\circ}$ and azimuth of $57^{\circ}$ with respect to a static user on earth. The interference source position is simulated to be at different azimuths and elevations from the user with a step size of $60^{\circ}$ in azimuth and $30^{\circ}$ in elevation, forming a grid of interference incident angles around PRN27. In addition to these angles, two more azimuth-elevation pairs that are close to PRN27, namely, $\left(30^{\circ}, 70^{\circ}\right)$ and $\left(40^{\circ}, 85^{\circ}\right)$, are simulated. Single PRN scenarios (for PRN27) are generated for each interference location separately and generated array data is processed using a blind eigenvector beamformer with seven taps, for each case. The overall measurement errors, $C / N_{0}$, and distortion metrics are measured for each case and are tabulated. The overall measurement errors are computed using (17) and the constant delay due to TDLs, namely, $\Delta \rho_{F}^{p}=104.93 \mathrm{~m}$, is removed. The measurement errors, $C / N_{0}$, and distortion metrics for all cases are given in Tables 4,5 , and 6 , respectively. It is evident from Tables 4 and 5 that when the interference is spatially away from the PRN, a very small bias gets added into the measurements and a high $C / N_{0}$ value is maintained. As the interference source approaches the satellite signal, the signal strength drops by a value of up to $6 \mathrm{~dB}$ and errors of up to $-132 \mathrm{~m}$ occur in the measurements. The errors and the $C / N_{0}$ variations as a function of interference location are shown in Figures 4 and 5. The satellite signal is shown as a circled star and the simulated positions of the interference sources are indicated using a plus symbol in the figures.

As discussed previously, asymmetrical widening of the CCF results in distortions. The amount of degradation depends on correlator spacing. To understand this behavior, 
TABLE 6: Changing interference incident angle: distortion metric (in meters).

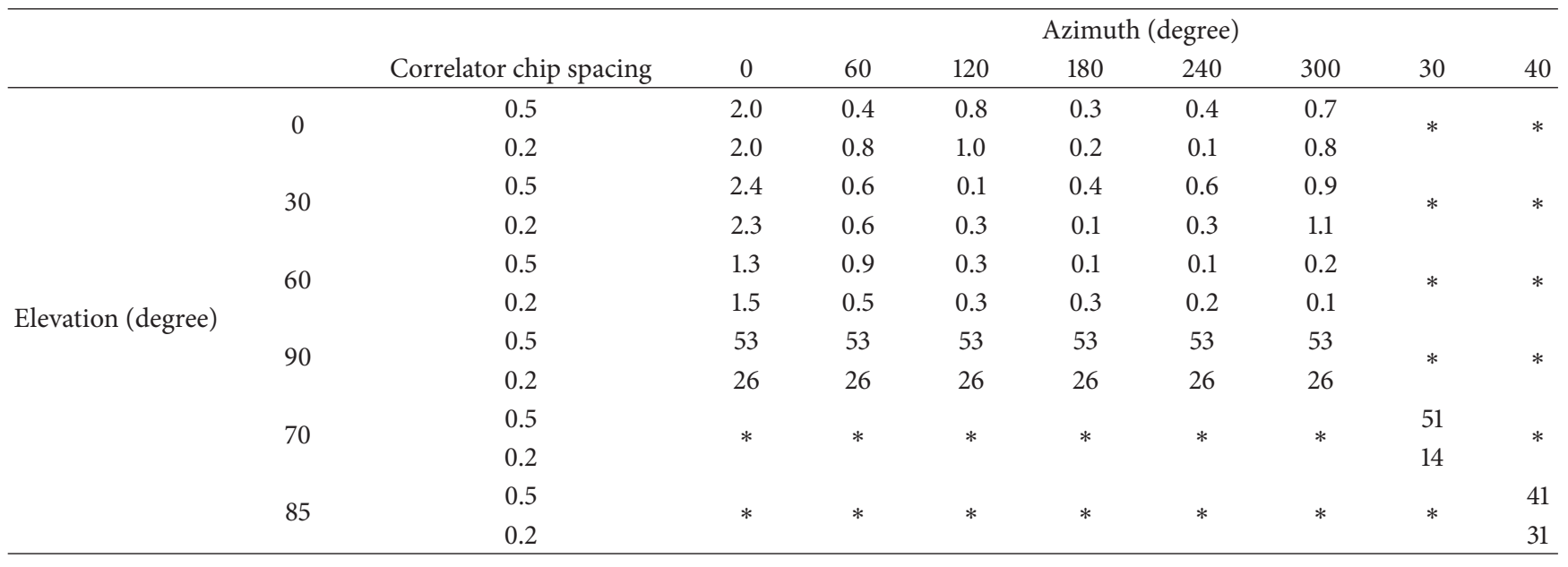

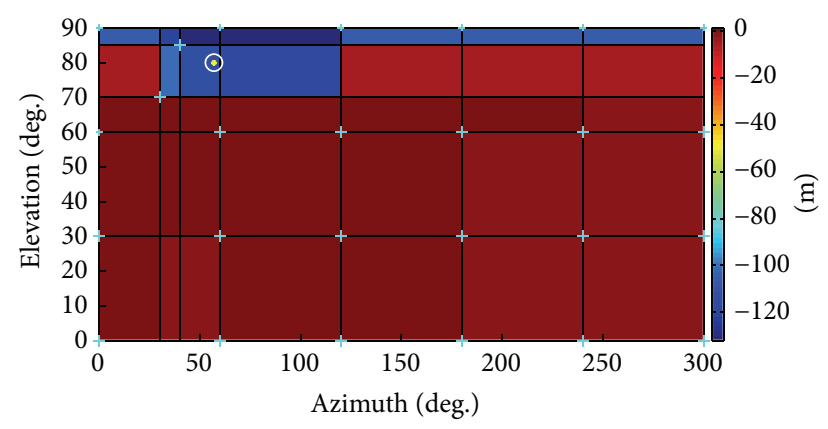

FIGURE 4: Changing interference incident angle-variation of $\Delta \rho_{V}^{p}$.

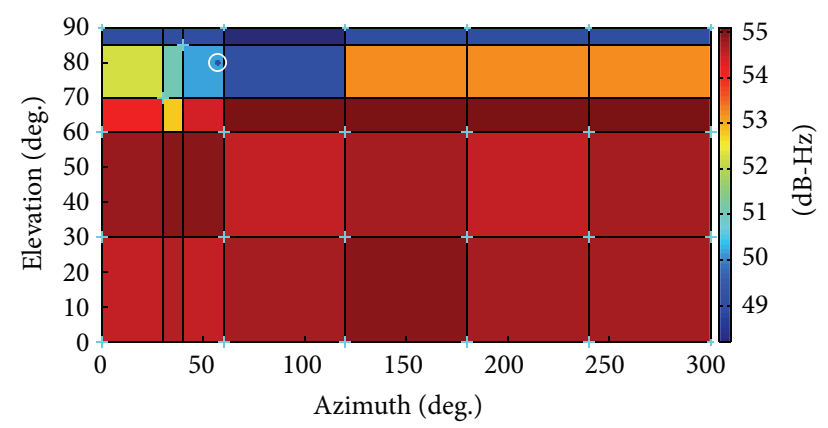

FIGURE 5: Changing interference incident angle-variation of $C / N_{0}$.

distortion metrics were measured for chip spacing of 0.2 and 0.5 and are given in Table 6 . Because of close proximity of the PRN and interference, errors up to $50 \mathrm{~m}$ are introduced due to CCF misshaping and the distortions were observed to be higher at 0.5 chip spacing compared to 0.2 chip spacing.

Based on the results obtained with the blind eigenvector method, it is apparent that the interference effects (as observed using $C / N_{0}$ and the errors) mostly do not change when the interference source is spatially away from the satellite signal. Therefore, for other beamforming methods, analysis of the interference placement effects is done only at a few sample points close to the desirable signal and results are given in Table 7. Results from the previous method are also added for the sake of comparison.

Since a single CW interference was used, MPDR introduced minimal distortions over a large space close to the interference with some variation in the signal strength. The E-MPDR provided an improved $C / N_{0}$ but higher distortion errors due to the temporal filter occur. As some DoF are consumed to maintain phase linearity in the C-DL method, smaller errors due to CCF misshaping were observed, compared to the blind method and E-MPDR. It can be seen that $C / N_{0}$ values for the C-DL method (distortionless STP) are lower compared to the E-MPDR method (semi-distortionless STP). This is owing to the fact that distortionless STP methods, in addition to steering the main lobe of the array beam pattern towards the direction of the received signals, apply a constraint to maintain the linearity of the filter phase response.

4.2. Effects of Different STP Distortions. Three different signal degradations that occur on GPS signals after performing STP are analyzed. The results obtained for different test cases are given here. These are categorized based on the distortion source in the following order:

(i) CCF Misshaping Only due to STP. This is analyzed using without noise scenarios generated for each PRN separately to avoid losses due to cross correlation. Six CW sources (as in Scenario 4 in Table 3) are simulated.

(ii) Effects of Noise and PRN's Cross Correlation. This is analyzed with multiple satellite scenarios with noise. Results are compared for one $\mathrm{CW}$ and six $\mathrm{CW}$ interference scenarios.

(iii) Effects of CCF Misshaping, Bias, Noise, and PRN's Cross Correlation. Combined effects due to above sources and the position error bias are given for one CW and six CW scenarios (Scenarios 2 and 4 in Table 3).

In order to characterize the effects of CCF misshaping and noise, one should have control to enable or disable noise in 
TABLE 7: Changing interference placement: comparison of different beamformers.

\begin{tabular}{|c|c|c|c|c|c|}
\hline \multirow{2}{*}{ (Azimuth, elevation) pair } & \multirow{2}{*}{ Parameter } & \multicolumn{4}{|c|}{ Beamforming method } \\
\hline & & Blind eigenvector & E-MPDR & $\mathrm{C}-\mathrm{DL}$ & MPDR \\
\hline \multirow{2}{*}{$(30,70)$} & $C / N_{0}(\mathrm{~dB}-\mathrm{Hz})$ & 51.1 & 52.3 & 50.8 & 51.4 \\
\hline & $\mathrm{DM}(\mathrm{m})$ & 51 & 26 & 3.9 & 0.2 \\
\hline \multirow{2}{*}{$(40,85)$} & $C / N_{0}(\mathrm{~dB}-\mathrm{Hz})$ & 49.0 & 51.8 & 47.8 & 45.8 \\
\hline & $\mathrm{DM}(\mathrm{m})$ & 41 & 6.2 & 3.0 & 1.0 \\
\hline \multirow{2}{*}{$(60,90)$} & $C / N_{0}(\mathrm{~dB}-\mathrm{Hz})$ & 49.5 & 52.2 & 50.7 & 50.8 \\
\hline & $\mathrm{DM}(\mathrm{m})$ & 53 & 11 & 2.6 & 1.5 \\
\hline
\end{tabular}

TABLE 8: Measurement errors using distortion metric without noise (six CW Scenario 4) (in meters).

\begin{tabular}{lcccc}
\hline \multirow{2}{*}{ SV ID } & \multicolumn{5}{c}{ Number of taps } \\
& 2 taps & 4 taps & 6 taps & 8 taps \\
\hline 8 & 43.2 & 20.4 & 8.1 & 5.2 \\
10 & 54.1 & 36.2 & 8.1 & 6.5 \\
17 & $*$ & 36.4 & 8.3 & 7.0 \\
18 & $*$ & $*$ & $*$ & 4.6 \\
27 & 60.5 & 19.3 & 9.0 & 5.9 \\
\hline
\end{tabular}

${ }^{*}$ Tracking with frequent loss of lock.

the generated signal. This is achieved by simulating signals without noise. Since the GPS is a Direct Sequence Code Division Multiple Access (CDMA) system, even if the effect of thermal noise is nullified during signal simulation, losses due to cross correlation between signals from different PRNs still persist. As a consequence, clean lossless GPS signals cannot be generated in a multisatellite environment. To address this, single PRN scenarios were individually generated for a few satellites. The proposed multiantenna simulation platform enables one to perform all the tests mentioned above and determine the contributions due to each part.

While generating the results, the deviations seen in the chip are translated to distance (as measured using distortion metric) and tabulated for comparison. An E-L correlator spacing of 0.2 chips is used in all experiments. These data sets are processed using a space-time filter adopting the blind eigenvector beamformer. The number of TDLs changes from 2 to 4,6 , and 8 for analyzing purpose.

CCF Misshaping Only due to STP. The CCF widening does not result in errors if widening occurs symmetrically but asymmetrical widening does. The interference scenario considered here contains six sources of interference. The single PRN scenarios for PRNs 8, 10, 17, 18, and 27 are simulated independently and the distortion metric used for the STP with different tap numbers is reported in Table 8 .

With a lower number of taps, complete signal recovery from interference could not be achieved for all PRNs and a frequent loss of tracking was observed. Interference mitigation was partially successful for 2 and 4 TDLs. By increasing the number of taps, better interference mitigation was observed which in turn led to improved acquisition and tracking. In spite of a widened CCF, an overall decrease in

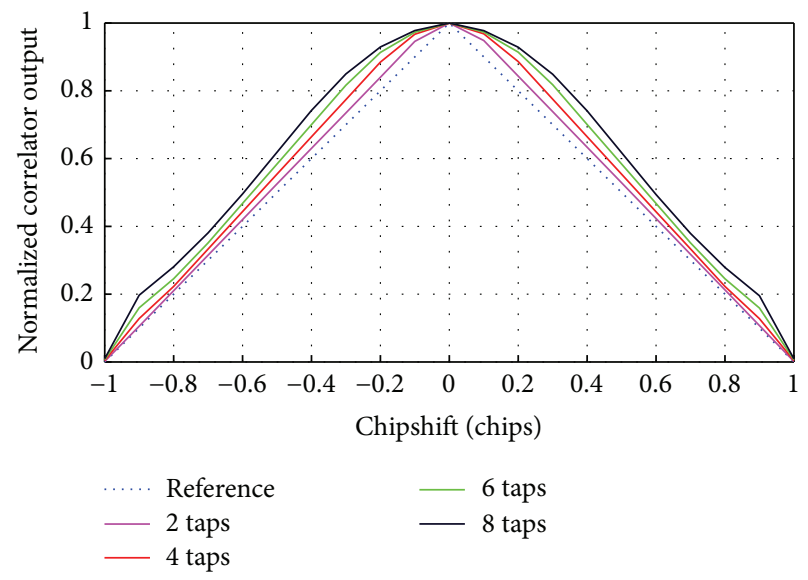

FIGURE 6: CCF distortions for PRN10-Scenario 2.

the distortion error was seen, due to improved mitigation performance.

Since the distortions are prominent even in the without noise case, it can be concluded that there is significant CCF distortion due to STP alone. A lesser distortion was observed with higher tap numbers; this could be due to an increase in the available temporal DoF in the space-time filter for interference mitigation. The CCF may get further distorted due to the presence of noise and cross correlation losses and these are analyzed further.

Effects of Noise and PRN's Cross Correlation. In this case, since signals for all PRNs are present, the noise floor increases due to the cross correlation. These are the most practical scenarios one encounters. Analysis is carried out on two multiple PRN scenarios, one with a single CW source (Interference Scenario 2) and another with six CW sources (Interference Scenario 4).

In the case of one CW, due to a favorable DoF, good quality interference mitigation is possible. Figure 6 shows a widening of the CCF with increasing taps for PRN10. The measurement errors due to the CCF distortion for all PRNs are reported in Table 9. Though not clearly conclusive, the trend observed suggests a distortion decrease at higher taps.

Similar distortion analysis was carried out for another multiple PRN scenario with comparatively intense interference conditions (Scenario 4). Due to the combined effect of PRN's cross correlation loss, noise, and STP, maximum impact is observed on the CCF distortion. The CCF for 
TABle 9: Measurement errors using distortion metric: for one CW and six CW (in meters).

\begin{tabular}{lccccc}
\hline \multirow{2}{*}{ SV ID } & \multirow{2}{*}{ Scenario } & \multicolumn{4}{c}{ Number of taps } \\
& & 2 taps & 4 taps & 6 taps & 8 taps \\
\hline \multirow{2}{*}{10} & 2 & 0.3 & 0.1 & 0.1 & 0.1 \\
& 4 & $*$ & $*$ & 27.7 & 26.2 \\
17 & 2 & 0.6 & 0.2 & 0.5 & 0.5 \\
& 4 & $*$ & $*$ & 26.1 & 22.5 \\
18 & 2 & 0.4 & 0.3 & 0.7 & 0.8 \\
& 4 & $*$ & $*$ & 22.1 & 26.6 \\
27 & 2 & 1.2 & 1.5 & 3.2 & 2.3 \\
& 4 & $*$ & $*$ & 32.9 & 23.1 \\
\hline
\end{tabular}

${ }^{*}$ Tracking with frequent loss of lock.

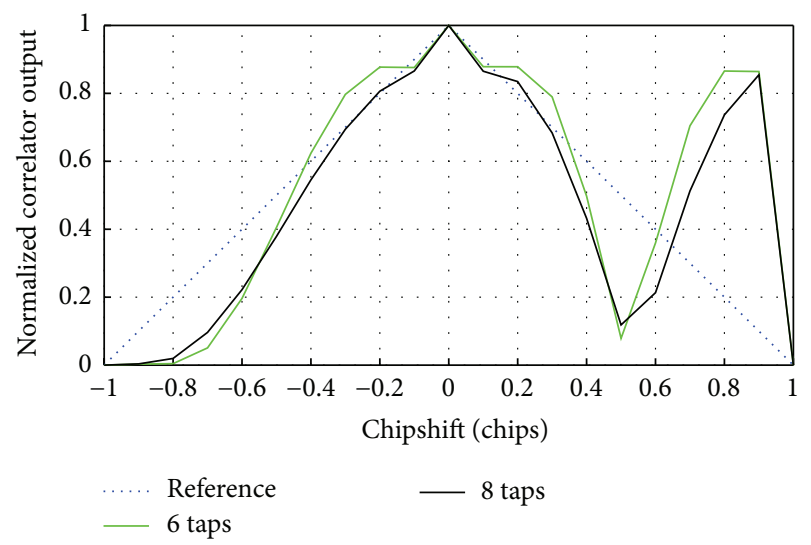

FIgURE 7: CCF distortions for PRN10-Scenario 4.

PRN10 is shown in Figure 7, where, with six CW, the CCF distortion is higher compared to that in the presence of one CW. Improved GPS signal recovery is seen with a higher number of taps. A five-to-seven-time increase in distortion errors is observed in the case of six CW, as compared to the one CW scenario case.

Effects of Noise and Cross Correlation, CCF Misshaping, and Bias. Here, the combined effect of all the distortion sources on the position errors is compared for different delays in the temporal filter of STP. The position accuracy of the receiver is determined by using the geometric DOP and RMS of measurement errors from all the satellites used for the position estimation [7]. The distortions present in each of the satellite measurements collectively contribute to an increase in position errors. Typically, the position errors in east (E), north $(\mathrm{N})$, and up (U) directions increase when the receiver antennas are exposed to a higher number of interferers as shown in Table 10.

Considering the previous discussions and looking at the position errors (for one CW scenario) given in Table 10, the measurement errors due to biases dominate the errors due to the CCF distortion and errors seem to increase as the number of taps increases. This leads to a position error increase (e.g., higher ENU errors for 8 taps compared to 6 taps in Scenario 2). Each addition of a TDL provides
TABLE 10: Position errors in the presence of distortions for different TDLs: for one CW and six CW (in meteres).

\begin{tabular}{ccccccc}
\hline & \multicolumn{3}{c}{ One CW (Scenario 2) } & \multicolumn{2}{c}{ Six CW (Scenario 4) } \\
& 2 taps & 4 taps & 6 taps & 8 taps & 6 taps & 8 taps \\
\hline E & -2.8 & -3.0 & -2.3 & -3.2 & -100 & -69 \\
$\mathrm{~N}$ & -1.8 & -1.9 & -1.5 & -2.1 & -64 & -29 \\
$\mathrm{U}$ & -1.1 & -1.1 & -0.9 & -1.2 & 38 & 21 \\
\hline
\end{tabular}

an increase in the number of temporal DoF available for interference mitigation. When the signal environment contains a higher number of interference sources, adding TDLs improves interference cancellation performance and in turn improves the acquisition and tracking behavior. For the specific case of six CW sources (Interference Scenario 4), partial success in acquisition/tracking of all PRNs was seen in the space-time filter with 2 and 4 TDLs and therefore position computation was not possible. The acquisition and tracking performance improved for 6 and 8 TDLs, increasing the number of satellites that can be used in the position computation. In Scenario 4, for the space-time filter with eight taps, an increase in the satellite count leads to a DOP improvement and in turn a reduction of position errors. The above observations suggest that the use of an optimal number of taps to achieve successful interference mitigation and at the same time maintain good position accuracy by maintaining less filter-biases is needed to achieve best results.

Effects of noise and cross correlation were studied for the blind eigenvector beamformer, as it is widely used in realworld applications due to fact that it does not require signal's AoA information compared to other methods considered in this research. Even though the results corresponding to noise analysis and varying number of taps are not presented and compared for other methods, one can expect a similar trend in results, possibly with different absolute values. In the following sections, distortions, biases, $C / N_{0}$, and position errors for different beamforming methods are compared for various simulated and live signal scenarios.

4.3. Comparison of Distortions in Beamformers. Distortions and their contributions in the position errors are analyzed for three different interference scenarios listed in Table 3. GPS signals are simulated as per the information of Table 2. Array data corresponding to these scenarios are processed using the four beamforming methods listed previously. For space-time processing six taps were used. Variable parts of the overall measurement error $\left(\Delta \rho_{V}^{p}\right)$ and DM are obtained for each beamformer and the results corresponding to PRN15 and PRN26 are reported in Table 11 for all scenarios.

The results of Table 11 show that DM values for blind STP are on the average higher for all scenarios. For the simple scenario (Scenario 2), DMs for all methods are mostly the same and have small magnitudes. The C-DL method reduces the CCF distortions in all scenarios. For the MPDR beamformer, since array data is collected from six elements, only five uncorrelated interference sources can be mitigated. Therefore, results are not obtained for Scenario 4. Nonequal 
TABLE 11: Distortions: for one CW, two CW + one WB, and six CW (in meters).

\begin{tabular}{|c|c|c|c|c|c|c|c|}
\hline \multirow{3}{*}{ Beamforming method } & \multirow{3}{*}{ Parameter } & \multirow{2}{*}{\multicolumn{2}{|c|}{$\begin{array}{c}\text { Scenario } 2 \\
\text { PRN }\end{array}$}} & \multirow{2}{*}{\multicolumn{2}{|c|}{$\begin{array}{c}\text { Scenario } 3 \\
\text { PRN }\end{array}$}} & \multirow{2}{*}{\multicolumn{2}{|c|}{$\begin{array}{c}\text { Scenario } 4 \\
\text { PRN }\end{array}$}} \\
\hline & & & & & & & \\
\hline & & 15 & 26 & 15 & 26 & 15 & 26 \\
\hline \multirow{2}{*}{ Blind eigenvector } & $\Delta \rho_{V}^{p}$ & 1.4 & -0.2 & -215 & -77 & 146 & -151 \\
\hline & $\mathrm{DM}$ & 1.8 & 0.5 & 53 & 1.7 & 6.7 & 5.8 \\
\hline \multirow{2}{*}{ MPDR } & $\Delta \rho_{V}^{p}$ & 0.3 & -0.3 & 0.3 & -0.4 & - & - \\
\hline & $\mathrm{DM}$ & 0.5 & 0.2 & 0.2 & 0.6 & - & - \\
\hline \multirow{2}{*}{ E-MPDR } & $\Delta \rho_{V}^{p}$ & 1.3 & 0.6 & 17 & 11 & 131 & 138 \\
\hline & $\mathrm{DM}$ & 3.1 & 2.6 & 20 & 14 & 25 & 21 \\
\hline \multirow{2}{*}{ C-DL } & $\Delta \rho_{V}^{p}$ & 0.6 & 0.2 & -2.6 & 1.9 & -3.3 & -4.2 \\
\hline & $\mathrm{DM}$ & 1.3 & 0.3 & 0.6 & 1.1 & 1.2 & 0.4 \\
\hline
\end{tabular}

TABLE 12: GPS position domain results for different methods and interference scenarios (for simulated GPS signals).

\begin{tabular}{|c|c|c|c|c|c|c|}
\hline \multirow{2}{*}{ Beamforming method } & \multirow{2}{*}{ Scenario } & \multicolumn{3}{|c|}{ Position errors $(\mathrm{m})$} & \multirow{2}{*}{$C / N_{0}(\mathrm{~dB}-\mathrm{Hz})$} & \multirow{2}{*}{ Tracking satellites } \\
\hline & & $\mathrm{E}$ & $\mathrm{N}$ & $\mathrm{U}$ & & \\
\hline \multirow{3}{*}{ Blind eigenvector } & 2 & -2.3 & -1.5 & -0.9 & 43.8 & 8 \\
\hline & 3 & -149 & -96 & -56 & 41.4 & 9 \\
\hline & 4 & 100 & 64 & 38 & 40.9 & 8 \\
\hline \multirow{3}{*}{ MPDR } & 2 & -3.6 & -2.3 & -1.3 & 49.7 & 9 \\
\hline & 3 & -2.7 & -1.7 & -1.0 & 47.1 & 9 \\
\hline & 4 & - & - & - & - & - \\
\hline \multirow{3}{*}{ E-MPDR } & 2 & -3.6 & -2.3 & -1.4 & 49.8 & 9 \\
\hline & 3 & -6.6 & -4.2 & -2.5 & 48.7 & 9 \\
\hline & 4 & 118 & 75 & 45 & 46.2 & 9 \\
\hline \multirow{3}{*}{ C-DL } & 2 & 0.7 & 0.4 & 0.3 & 47.3 & 9 \\
\hline & 3 & -6.5 & -4.2 & -2.4 & 39.9 & 8 \\
\hline & 4 & -19 & -12 & -7.0 & 40.2 & 9 \\
\hline
\end{tabular}

measurement biases can be observed from the $\Delta \rho_{V}^{p}$ values recorded in Table 11. Measurement biases increase for all methods with an increasing number of interferences. In addition to the reduction of DM values, the C-DL method results in smaller biases. Even though there are only three interference sources in Scenario 3, significant biases are observed. This could be due to the presence of wideband interference, which generally consumes more DoF during mitigation than CW interference.

Table 12 lists position errors in the ENU coordinate system for all three scenarios after employing the beamformers described in Section 3. For Scenario 2, the position error magnitude is almost the same for the different beamformers. The blind eigenvector method provides an accurate position for the mild interference scenario and large position errors for harsh scenarios. The MPDR beamformer is only based on spatial processing and CCFs and position solutions do not experience any distortion due to time filtering. Results in Table 12 verify the fact that the MPDR beamformer can suppress one interference (Scenario 2) and three interference sources (Scenario 3) without generating significant ENU errors but is not able to mitigate six uncorrelated narrowband interference signals (Scenario 4). The E-MPDR method successfully mitigates six interference sources as a result of additional DoF from temporal filters but introduces some bias, leading to position estimates that are inferior to MPDR. Results show that the C-DL method not only provides extra DoF for narrowband interference mitigation compared to MPDR but also keeps the CCFs less distorted and measurements less biased. Therefore its positioning performance is considerably better than that of the other STP methods. Similar to the observations made in Section 4.1, $C / N_{0}$ values for the C-DL method are lower compared to corresponding MPDR and E-MPDR values. The $C / N_{0}$ values also indicate the gain achieved (or the losses incurred) during array processing. This gain can be analyzed using antenna array gain patterns. Considering (10), for a space-time filter the gain pattern (in $\mathrm{dB}$ ) is calculated as $10 \log \left(\left|\mathbf{h}^{H}(f) \mathbf{a}_{p}\right|^{2}\right)$, where $\mathbf{h}^{H}(f) \mathbf{a}_{p}$ is the response of the filter to the input signals with the steering vector $\mathbf{a}_{p}$ at frequency $f$. Array gain pattern is a function of frequency and signal's AoA.

Array gain patterns for the four methods mentioned in Table 11 are shown in Figure 8 for Scenario 2. As previously mentioned a single set of filter weights is computed in the blind eigenvector beamformer array gain pattern as shown in Figure 8(a). The inability of the blind method to provide sustained gain for all satellites (indicated with a tag "S" in Figure 8) is evident. The MPDR, E-MPDR, and C-DL methods use signal's AoA; therefore illustrations are provided for only one satellite PRN (i.e., PRN8, marked in 


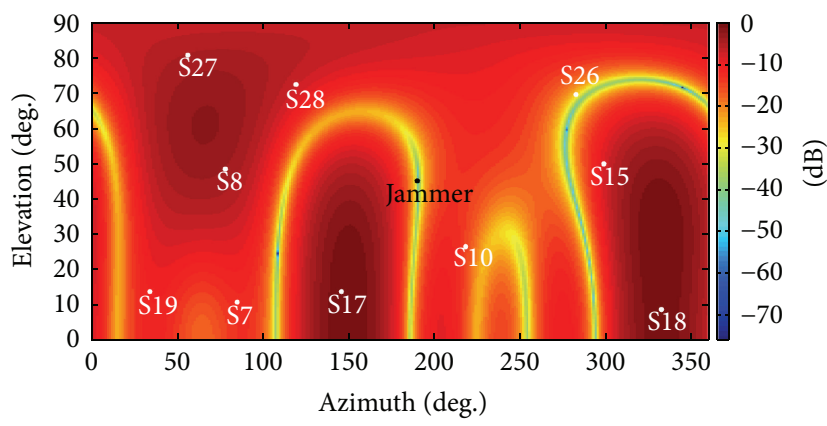

(a) Blind eigenvector

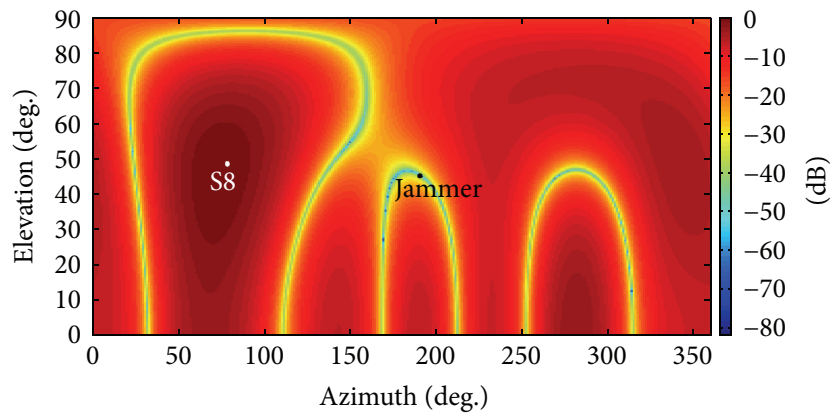

(c) E-MPDR

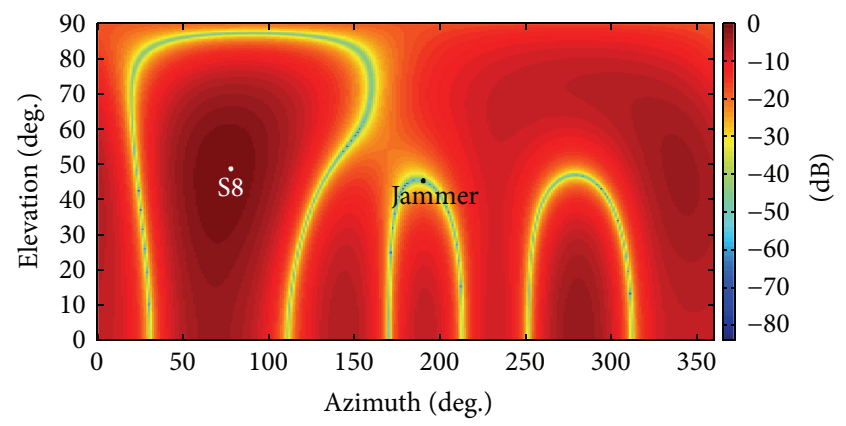

(b) MPDR

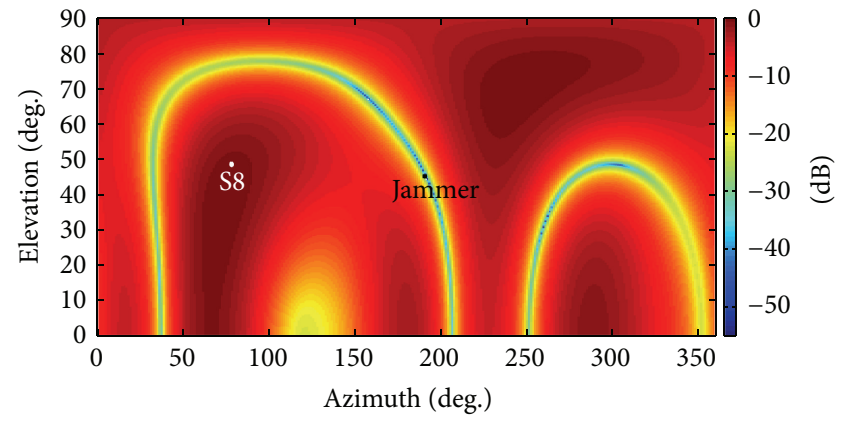

(d) C-DL

FIGURE 8: Normalized antenna array gain patterns at the interference frequency for different methods (PRN8-Scenario 2).

the figure as S8). The MPDR and E-MPDR exhibit similar beam patterns as shown in Figures 8(b) and 8(c). However, there is an increased depth of the nulls in the interference direction in E-MPDR for a few satellites. Shallow nulls in the interference direction are seen in C-DL (Figure 8(d)) and these can potentially cause a drop in $C / N_{0}$ values compared to other methods (as observed in Table 12).

\section{Experimental Results and Analyses}

The performance evaluation and comparison of the measurement distortions with different beamforming methods were done in the previous sections using simulated scenarios. Real data tests are now discussed.

5.1. Data Collection Setup and Processing. Due to transmission regulations, interference was generated in software and added to digitized live GPS data samples collected using an antenna array. The data collection environment and test setup are shown in Figure 9. Intermediate frequency (IF) samples for GPS L1 C/A signals were collected using a six-element antenna array. Data was collected in a parking area with clear sky conditions for satellite visibility. The vehicle was moved in a circular trajectory to receive signals from various directions for the calibration process (Figure $9(\mathrm{a})$ ). The antenna array was mounted on the vehicle top (Figure 9(b)) and the RF cables from the six antenna elements were connected to the phase coherent six-channel Fraunhofer/TeleOrbit RF frontend (Figure 9(c)). The received signals were then downconverted, digitized, and stored for postprocessing (Figure 9(d)).
TABLE 13: Satellite visibility during test: real data.

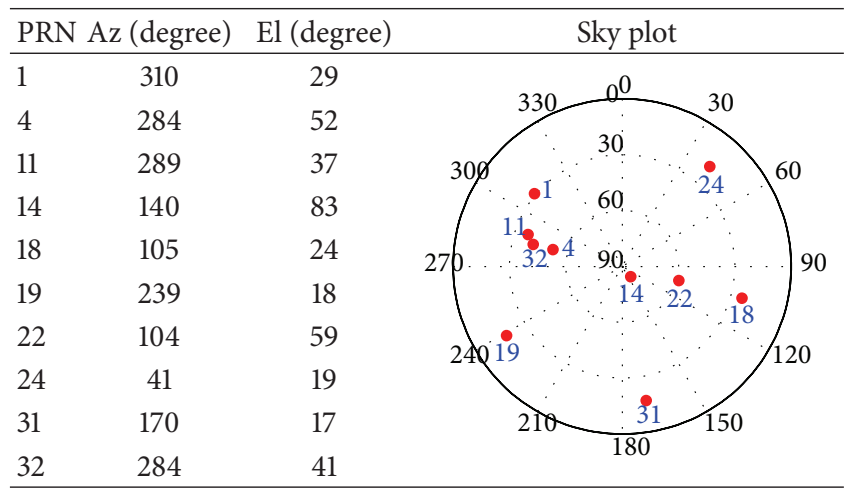

A sampling frequency of $20 \mathrm{MHz}$ was used. The reference position was obtained using a NovAtel SPAN ${ }^{\mathrm{TM}}$ LCI system based differential positioning method and was accurate to a few centimeters in open-sky conditions [38].

The PRN codes and azimuth and elevation angles of the satellites visible during data collection are given in Table 13; 10 GPS satellites were available.

The antenna array used for interference mitigation consists of antenna elements placed close to each other. When antennas are placed in the near field of each other the amplitude and phase of the received signals at each element may vary. In addition, the amplitude and phase response at each element might be affected due to mutual coupling, differing cable lengths, and antenna phase centre variations. This necessitates array calibration in many GNSS applications 
TABLE 14: GPS position domain results for different methods and interference scenarios (for live GPS signals)* .

\begin{tabular}{|c|c|c|c|c|c|c|}
\hline \multirow{2}{*}{ Beamforming method } & \multirow{2}{*}{ Scenario } & \multicolumn{3}{|c|}{ Position errors (m) } & \multirow{2}{*}{$C / N_{0}(\mathrm{~dB}-\mathrm{Hz})$} & \multirow{2}{*}{ Tracking satellites } \\
\hline & & $\mathrm{E}$ & $\mathrm{N}$ & $\mathrm{U}$ & & \\
\hline \multirow{2}{*}{ Blind eigenvector } & 2 & -1.6 & 1.6 & -0.3 & 47.9 & 9 \\
\hline & 4 & 621 & 4688 & 2021 & 40.9 & 7 \\
\hline \multirow{2}{*}{ MPDR } & 2 & -1.6 & 0.6 & 2.7 & 51.5 & 10 \\
\hline & 4 & - & - & - & - & - \\
\hline \multirow{2}{*}{ E-MPDR } & 2 & -1.6 & 0.4 & 3.0 & 51.7 & 10 \\
\hline & 4 & 42 & -143 & -160 & 43.8 & 10 \\
\hline \multirow{2}{*}{$\mathrm{C}-\mathrm{DL}$} & 2 & -1.9 & 1.1 & 4.2 & 48.6 & 10 \\
\hline & 4 & -45 & -133 & 42 & 39.1 & 8 \\
\hline
\end{tabular}

${ }^{*}$ For $60 \mathrm{~s}$ of data in the static mode.

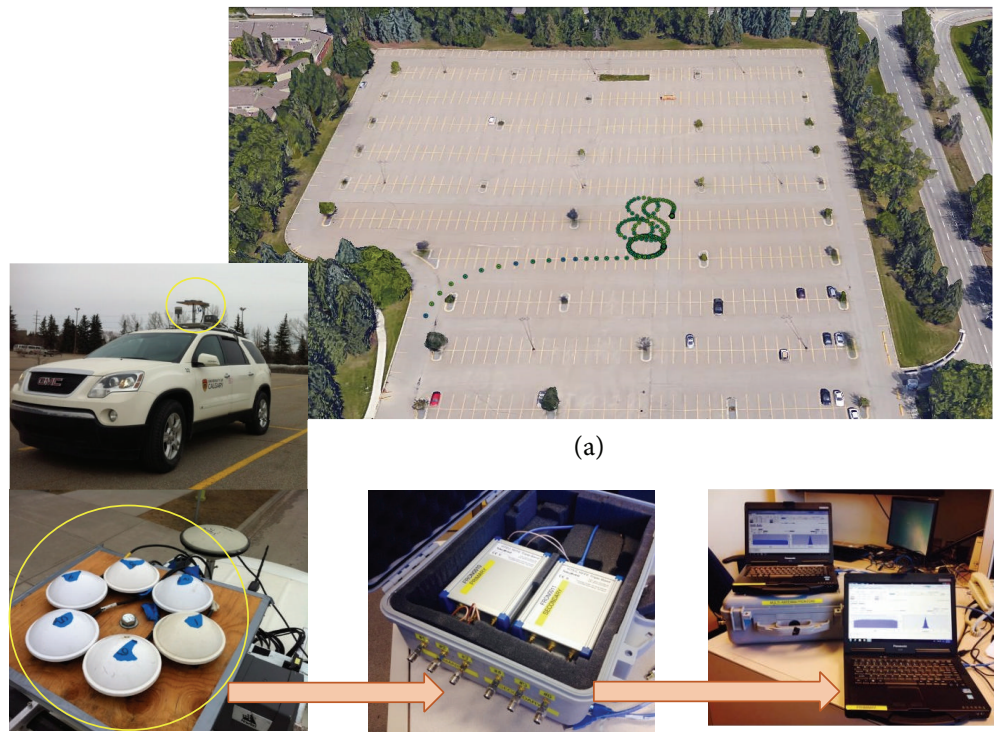

(b) (d)

FigURE 9: Data collection scenario and setup.

employing spatial processing, in order to steer nulls towards undesired signal directions while maintaining the main lobe of the beam pattern in the direction of the desired signal.

Antenna arrays are commonly calibrated using anechoic chambers by scanning all incident signals from different AoA values [39]. When antenna arrays are used in GPS, the GPS signals can themselves be used as radio frequency sources with known AoA. This eliminates the need for expensive anechoic chambers. An on-site calibration approach proposed in [40] was used. As discussed in Section 3.1, steering vectors model the phase offsets between each antenna element and the reference element. However, in general, as the beamforming methods cannot compensate the signal delays between the array elements in the pseudoranges, a small contribution to biases can be present in the results. Contributions from these biases should be noticed while comparing the real data results against the simulated results, as these biases are absent in the latter case.
5.2. Distortion Analysis Using Position Errors and $C / N_{0}$ Value. When live data is collected, there can be additional contributors to the CCF distortion. The multipath signals arriving at the front-end might add up (constructively or destructively) and distort the CCF, in addition to the other sources described earlier. Here, only position errors and $C / N_{0}$ are analyzed for live scenarios and are given in Table 14. Simple and harsh scenarios are considered for live data results and the focus is to verify the results with those from the simulations discussed in the previous sections. Meanwhile, one can observe the contributions from additional measurement errors, for example, errors due to atmospheric delays and multipath (if any).

Conclusions similar to those of the simulated data can be drawn. The magnitudes of position errors in Table 14 are higher than the errors in Table 12. These are likely due to the errors present in the signals before array processing and calibration errors. Improved SNR performance occurs with 
the E-MPDR method and the SNR performance of the C-DL method is inferior due to the possible usage of some array DoF for maintaining phase linearity; however, C-DL provides good position performance.

\section{Summary and Conclusions}

A theoretical analysis of the distortions observed in the GPS measurements due to antenna array processing was provided and supported with results from simulated and live signals, for different beamforming methods categorized as blind, semi-distortionless, and distortionless. A distortion metric and a measure of overall bias that would help to quantify the distortions in space-time processing were introduced. The effects of the placement of the interference source related to the correct signal direction were shown using overall measurement errors and a profile of the signal strength for different methods. The use of single PRN noise free signal simulations to study CCF distortions showed that STP contributes significantly to CCF distortion. In the presence of a higher number of interference sources, mitigation performance improvement was observed when increasing the number of taps. The distortions and their contributions in the position errors were characterized for simulated signals and real data using a real antenna array. It can be concluded that STP borne distortions lead to erroneous pseudorange measurements and degrade GNSS position accuracy performance in challenging environments.

Selection of a proper method depends on the interference scenario. In a simple scenario, the number of acquired satellites, $C / N_{0}$ values, and position accuracy are excellent for all methods. The blind beamformer is a better choice for some low level scenarios, given its low complexity. In the blind method, since AoA are not used and the method does not involve modifications to the standard receiver operation, the method is independent of the receiver structure; therefore, an antenna array followed by the blind method can be used as a replacement for the antenna of any single antenna receiver. The method is not however a good choice for harsh interference scenarios or high accuracy applications because the number of acquired satellites and position accuracy are low. In harsh environments, the semi-distortionless (E-MPDR) or distortionless (C-DL) methods are better choices. They both have almost the same amount of complexity (the distortionless complexity is slightly higher). The distortionless method provides a higher accuracy, whereas the semi-distortionless method results in a higher number of satellites acquired and $C / N_{0}$. As long as sufficient satellites track with a good signal strength, the distortionless method is a better choice; if the number of satellites is reduced or $C / N_{0}$ decreases, then the semi-distortionless method is better. Therefore, an optimum receiver should switch between these two cases depending on the situation.

\section{Conflict of Interests}

The authors declare that there is no conflict of interests regarding the publication of this paper.

\section{References}

[1] B. Bonet, I. Alcantarilla, D. Flament, C. Rodríguez, and N. Zarraoa, "The benefits of multi-constellation GNSS: reaching up even to single constellation GNSS users," in Proceedings of the 22nd International Technical Meeting of the Satellite Division of the Institute of Navigation (ION GNSS '09), pp. 1268-1280, Savannah, Ga, USA, September 2009.

[2] S. De Lorenzo, F. Antreich, H. Denks, A. Hornbostel, C. Weber, and P. Enge, "Testing of adaptive beamsteering for interference rejection in GNSS receivers," in Proceedings of the European Navigation Conference (ENC-GNSS '07), Geneva, Switzerland, May-June 2007.

[3] D. Borio, "Interference detection and mitigation," in Proceedings of the ESA International Summer School on GNSS, European Commission Joint Research Centre, ESA, Davos, Switzerland, July 2013, http://www.danieleborio.altervista.org/sumschool/ EsaSc_Interference_BorioD_07Jun13.pdf.

[4] J. R. Sklar, "Interference mitigation approaches for the global positioning system," Lincoln Laboratory Journal, vol. 14, no. 2, pp. 167-180, 2003.

[5] C. Church, I. Gupta, and A. O’Brien, "Adaptive antenna induced biases in GNSS receivers," in Proceedings of the 63rd Annual Meeting of the Institute of Navigation, pp. 204-212, Cambridge, Mass, USA, April 2007.

[6] E. D. Kaplan and C. J. Hegarty, Understanding GPS-Principles and Applications, Artech House, Norwood, Mass, USA, 2006.

[7] P. Misra and P. Enge, Global Positioning System-Signals, Measurements, and Performance, Ganga-Jamuna Press, Lincoln, Neb, USA, 2001.

[8] D. S. De Lorenzo, S. C. Lo, P. K. Enge, and J. Rife, "Calibrating adaptive antenna arrays for high-integrity GPS," GPS Solutions, vol. 16, no. 2, pp. 221-230, 2012.

[9] L. Shuangxun, C. Zhu, H. Kan, and X. Hongyin, "A compensating approach for signal distortion introduced by STAP," in Proceedings of the International Conference on Communication Technology (ICCT '06), pp. 1-4, IEEE, Guilin, China, 2006.

[10] R. L. Fante and J. J. Vaccaro, "Wideband cancellation of interference in a GPS receive array," IEEE Transactions on Aerospace and Electronic Systems, vol. 36, no. 2, pp. 549-564, 2000.

[11] G. F. Hatke, "Adaptive array processing for wideband nulling in GPS systems," in Proceedings of the 32nd Asilomar Conference on Signals, Systems \& Computers, vol. 2, pp. 1332-1336, IEEE, Pacific Grove, Calif, USA, November 1998.

[12] W. L. Myrick, M. D. Zoltowski, and S. J. Goldstein, "Exploiting conjugate symmetry in power minimization based preprocessing for GPS: reduced complexity and smoothness," in Proceedings of the International Conference on Acoustics, Speech, and Signal Processing, pp. 2833-2836, IEEE, Istanbul, Turkey, June 2000.

[13] W. L. Myrick, G. J. Scott, and M. D. Zoltowski, "Low complexity anti-jam space-time processing for GPS," in Proceedings of the IEEE International Conference on Acoustics, Speech, and Signal Processing (ICASSP '01), pp. 2233-2236, IEEE, Salt Lake City, Utah, USA, May 2001.

[14] B. D. Van Veen and K. M. Buckley, "Beamforming: a versatile approach to spatial filtering," IEEE ASSP Magazine, vol. 5, pp. 4-24, 1988. 
[15] S. Daneshmand, A. J. Jahromi, A. Broumandan, and G. Lachapelle, "GNSS space-time interference mitigation: advantages and challenges," in Proceedings of the International Symposium on GNSS (IS-GNSS '15), p. 11, Kyoto, Japan, November 2015.

[16] A. Konovaltsev, D. S. De Lorenzo, A. Hornbostel, and P. Enge, "Mitigation of continuous and pulsed radio interference with GNSS antenna arrays," in Proceedings of the 21st International Technical Meeting of the Satellite Division of the Institute of Navigation (ION GNSS '08), pp. 2786-2795, Savannah, Ga, USA, September 2008.

[17] A. J. O’Brien and I. J. Gupta, "Mitigation of adaptive antenna induced bias errors in GNSS receivers," IEEE Transactions on Aerospace and Electronic Systems, vol. 47, no. 1, pp. 524-538, 2011.

[18] S. Daneshmand, A. J. Jahromi, A. Broumandan, and G. Lachapelle, "GNSS space-time interference mitigation and attitude determination in the presence of interference signals," Sensors, vol. 15, no. 6, pp. 12180-12204, 2015.

[19] R. L. Fante, M. P. Fitzgibbons, and K. F. McDonald, "Effect of adaptive array processing on GPS signal crosscorrelation," in Proceedings of the 17th International Technical Meeting of the Satellite Division of the Institute of Navigation (ION GNSS '04), pp. 579-583, Long Beach, Calif, USA, September 2004.

[20] Y. C. Chuang and I. J. Gupta, "Antenna induced biases in GNSS receiver measurements," in Proceedings of the Institute of Navigation International Technical Meeting (ITM '13), pp. 164171, San Diego, Calif, USA, January 2013.

[21] G. Heinrichs, E. Loehnert, and E. Wittmann, "User RAIM integrity and interference mitigation test results with upgraded German Galileo test range GATE," in Proceedings of the 5th ESA Workshop on Satellite Navigation Technologies and European Workshop on GNSS Signals and Signal Processing (NAVITEC '10), pp. 1-7, IEEE, Noordwijk, The Netherlands, December 2010.

[22] L. Dong, IF GPS signal simulator development and verification [M.S. thesis], Department of Geomatics, University of Calgary, Calgary, Canada, 2003, http://plan.geomatics.ucalgary.ca/ papers/03.20184.leidong.pdf.

[23] W. Y. Zhao, L. F. Xu, and R. B. Wu, "A simulation tool for space-time adaptive processing in GPS," in Proceedings of the Progress in Electromagnetics Research Symposium, Cambridge, Mass, USA, March 2006.

[24] Y. Zheng, Adaptive antenna array processing for GPS receivers [M.S. thesis], School of Electrical \& Electronics Engineering, University of Adelaide, Adelaide, Australia, 2008, https://digital .library.adelaide.edu.au/dspace/bitstream/2440/49670/8/02whole.pdf.

[25] Spirent Communications PLC, Simgen Software User Manual, Spirent Communications PLC, Paignton, UK, 2012.

[26] H. L. Van Trees, Optimum Array Processing-Part IV of Detection, Estimation, and Modulation Theory, John Wiley \& Sons, New York, NY, USA, 2002.

[27] Rohde \& Schwarz, Satellite Navigation Digital Standards for ReS SMBV-Operating Manual, Mühldorfstr, Munich, Germany, 2014.

[28] R. A. Monzingo, R. L. Haupt, and T. W. Miller, Introduction to Adaptive Arrays, SciTech Publishing, Raleigh, NC, USA, 2011.

[29] T. K. Citron and T. Kailath, "An improved eigenvector beamformer," in Proceedings of the IEEE International Conference on Acoustics, Speech, and Signal Processing (ICASSP '84), pp. 718721, IEEE, San Diego, Calif, USA, March 1984.
[30] T. Marathe, S. Daneshmand, and G. Lachapelle, "Pseudolite interference mitigation and signal enhancements using an antenna array," in Proceedings of the International Conference on Indoor Positioning and Indoor Navigation (IPIN '15), pp. 19, Banff, Canada, October 2015.

[31] P. B. Anantharamu, D. Borio, and G. Lachapelle, "Space-time equalization techniques for new GNSS signals," in Proceedings of the 24th International Technical Meeting of the Satellite Division, US Institute of Navigation, Portland, Ore, USA, September 2011.

[32] J. Bao and Y. Tsui, Fundamentals of Global Positioning System Receivers-A Software Approach, John Wiley \& Sons, Hoboken, NJ, USA, 2000.

[33] M. Abdizadeh, GNSS signal acquisition in the presence of narrowband interference [Ph.D. thesis], Department of Geomatics Engineering, University of Calgary, Calgary, Canada, 2013, http://plan.geomatics.ucalgary.ca/.

[34] Y. Peng, Z. Deng, G. Fan, and P. Chen, “Time domain filtering based compensation technique for space-time adaptive processing," in Proceedings of the China Satellite Navigation Conference (CSNC, '12), vol. 161, pp. 559-569, Springer, Guanzhou, China, May 2012.

[35] T. Marathe, S. Daneshmand, and G. Lachapelle, "Characterizing signal distortion due to space-time processing of interference impacted GNSS signals," in Proceedings of the ION GNSS+2015, The Institute of Navigation, Tampa, Fla, USA, September 2015.

[36] K. Borre, D. M. Akos, N. Bertelsen, P. Rinder, and S. H. Jensen, A Software-Defined GPS and Galileo Receiver: A Single-Frequency Approach, Springer, Birkhäuser, Boston, Mass, USA, 2007.

[37] J. W. Betz, "Effect of partial-band interference on receiver estimation of C/N0: theory," in Proceedings of the National Technical Meeting of the Institute of Navigation (ION-NTM '01), pp. 817-828, Long Beach, Calif, USA, January 2001.

[38] Novatel, 2015, http://www.novatel.com/.

[39] U. S. Kim, D. De Lorenzo, J. Gautier, P. Enge, and J. A. Orr, "Phase effects analysis of patch antenna CRPAs for JPALS," in Proceedings of the 17th International Technical Meeting of the Satellite Division (ION GNSS '04), pp. 1531-1538, The Institute of Navigation, Long Beach, Calif, USA, September 2004.

[40] S. Daneshmand, N. Sokhandan, M. Zaeri-Amirani, and G. Lachapelle, "Precise calibration of a GNSS antenna array for adaptive beamforming applications," Sensors, vol. 14, no. 6, pp. 9669-9691, 2014. 


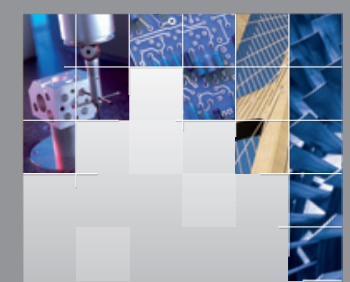

\section{Enfincering}
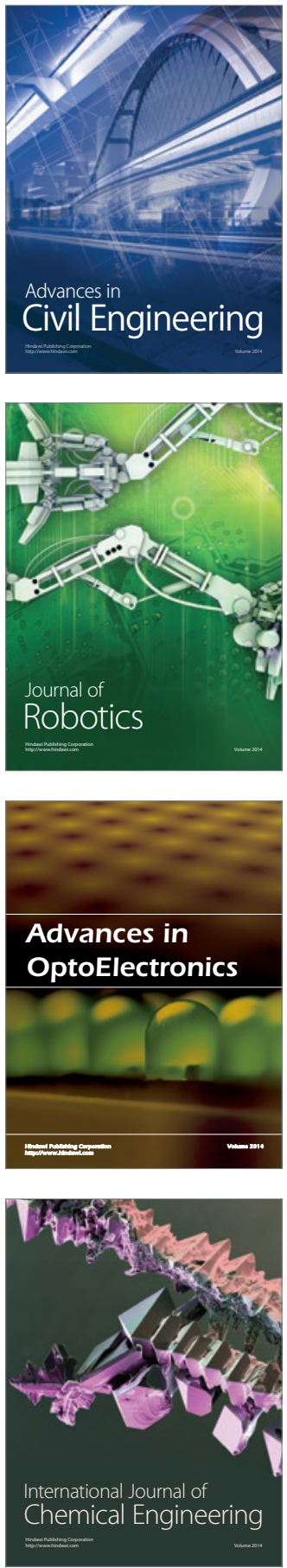

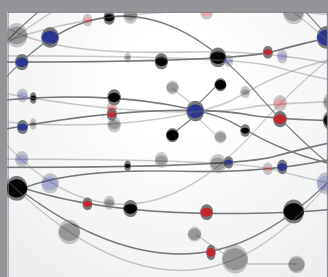

The Scientific World Journal

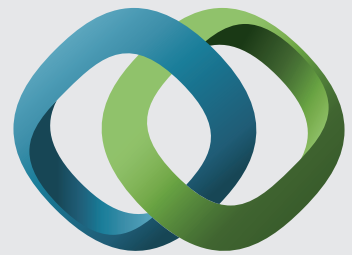

\section{Hindawi}

Submit your manuscripts at

http://www.hindawi.com
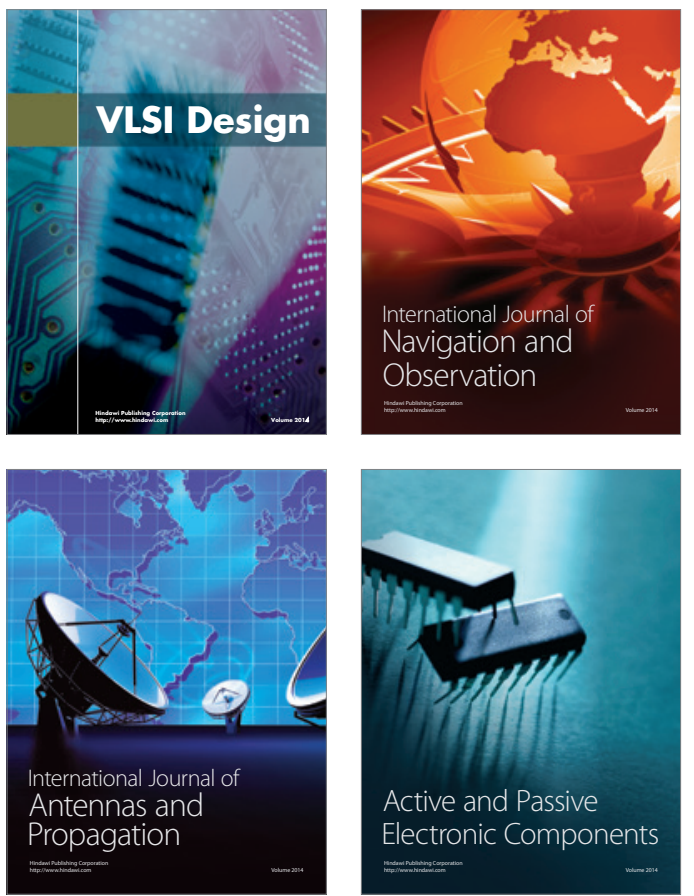
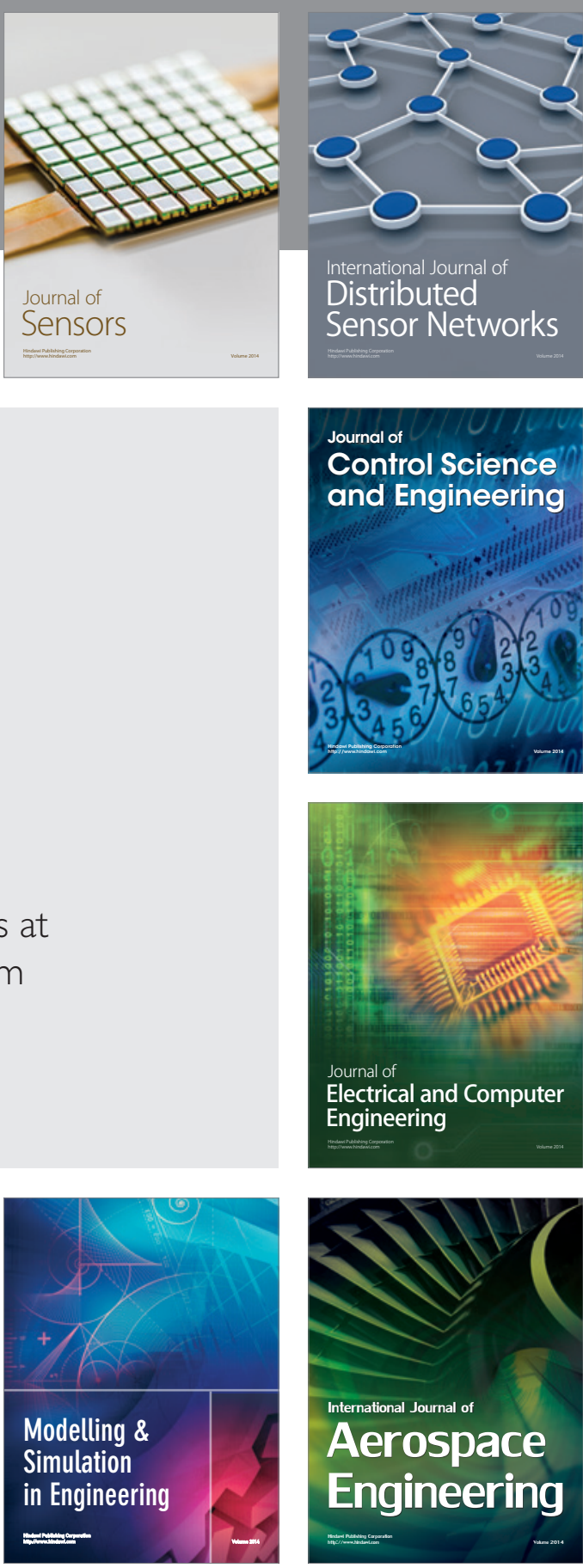

International Journal of

Distributed

Sensor Networks

Journal of

Control Science

and Engineering
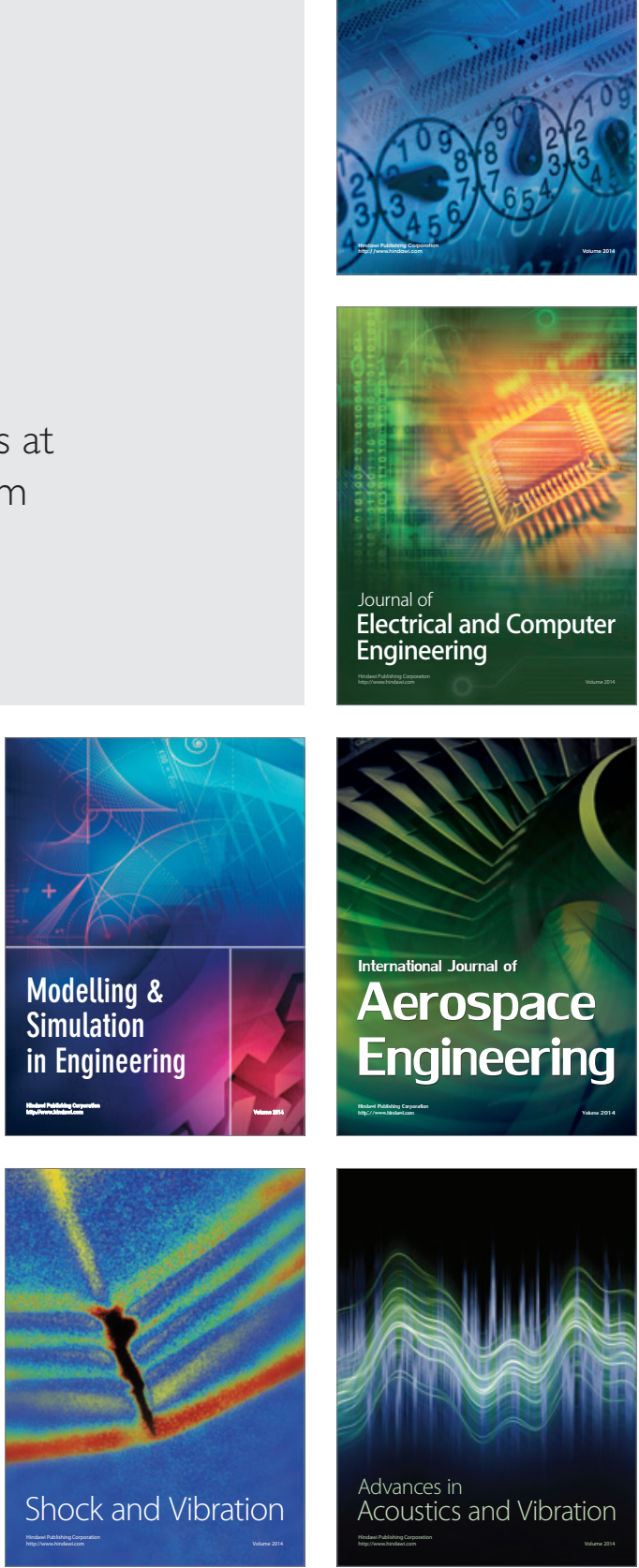